\title{
A quantum prediction as a collection of epistemically restricted classical predictions
}

\author{
William F. Braasch Jr. ${ }^{1}$ and William K. Wootters ${ }^{2}$ \\ ${ }^{1}$ Department of Physics and Astronomy, Dartmouth College, Hanover, New Hampshire 03755, USA \\ ${ }^{2}$ Department of Physics, Williams College, Williamstown, Massachusetts 01267, USA
}

Spekkens has introduced an epistemically restricted classical theory of discrete systems, based on discrete phase space. The theory manifests a number of quantum-like properties but cannot fully imitate quantum theory because it is noncontextual. In this paper we show how, for a certain class of quantum systems, the quantum description of an experiment can be decomposed into classical descriptions that are epistemically restricted, though in a different sense than in Spekkens' work. For each aspect of the experiment - the preparation, the transformations, and the measurement-the epistemic restriction limits the form of the probability distribution an imagined classical observer may use. There are also global constraints that the whole collection of classical descriptions must satisfy. Each classical description generates its own prediction regarding the outcome of the experiment. One recovers the quantum prediction via a simple but highly nonclassical rule: the "nonrandom part" of the predicted quantum probabilities is obtained by summing the nonrandom parts of the classically predicted probabilities. By "nonrandom part" we mean the deviation from complete randomness, that is, from what one would expect upon measuring the fully mixed state.

\section{Introduction}

For any physical theory, it is generally a good thing to have a number of different formulations of the theory. Alternative formulations can provide novel insights and can offer practical problem-solving tools, and if the theory ultimately needs to be modified, the needed modification might be more easily identified within one formulation than within others.

William F. Braasch Jr.: William.Frederick.Braasch.Jr@dartmouth.edu William K. Wootters: wwootter@williams.edu
The ultimate goal motivating the present work is to produce an alternative formulation of quantum theory, based on phase space rather than Hilbert space, and, unlike the Wigner-function formulation, based on actual probability distributions over phase space rather than on quasiprobability distributions (that is, distributions that can take negative values). Although we do not achieve this aim in this paper, we do succeed in re-expressing in these terms the quantum theory of systems that, in the Hilbert-space formulation, have oddprime-power Hilbert-space dimensions. We discuss in Section 8 the particular challenges involved in extending our work to other dimensions.

Part of the impetus for our work is a 2016 paper by Spekkens based on unpublished work from 2008 [1-3]. Spekkens presents an epistemically restricted classical theory of discrete systems and shows that this theory exhibits a remarkable number of quantum-like features. By construction, however, the theory is noncontextual and can therefore not fully imitate quantum theory. It is also thoroughly discrete, with only finitely many pure states for any given system. An extention of Spekkens' model designed to allow for contextuality has been introduced but is limited to the setting of a pair of toy bits [4]. In the present paper, we show how a greater part of quantum theory can be re-expressed in terms of classical descriptions that are likewise epistemically restricted, though, as we explain, our restrictions are more specific than those in Spekkens' model. In addition to potentially leading to a full alternative formulation of quantum theory, we feel that this construction provides a novel perspective on the relation between classical and quantum physics.

Let us use the word "experiment" to refer to a sequence of operations consisting of a preparation, a transformation (or possibly a sequence of transformations), and a measurement. In Spekkens' approach, an experiment performed on an elementary discrete system is described in terms of a discrete phase space that can be pictured as a $d \times d$ array of points, where $d$ is a prime number. The points of this phase space are understood to be the actual physical states of the system - the "on- 
tic states." But an epistemic restriction prevents an observer from knowing the current ontic state: the most they can know is that the system's state lies on a particular line in the phase space. The allowed transformations in Spekkens' picture are the affine-symplectic transformations, which are analogous to certain allowed classical transformations in continuous phase space. Finally, the finest-grained type of measurement allowed is a measurement that distinguishes the lines constituting a complete set of parallel lines, which we call a "striation" of the phase space. Distinguishing one line in such a set is analogous to measuring, say, the position of a particle while not simultaneously measuring its momentum or any nontrivial linear combination of position and momentum. (Each of these observables is associated with a set of parallel lines in phase space having a particular slope.)

In the present paper, we similarly consider epistemically restricted classical accounts based on a $d \times d$ phase space with prime $d$. (We later extend the formalism to odd prime powers.) Specifically, we show how to decompose the description of a quantum experiment into a collection of classical descriptions, each of which adheres to certain epistemic restrictions. As it turns out, these descriptions are also interdependent in that they must satisfy a set of global constraints, which correspond to the constraints on the mathematical objects appearing in the standard Hilbert space formulation of quantum mechanics.

Each of the classical descriptions of an experiment will generate its own prediction regarding the outcome: it generates a probability that any particular event will occur. Much of our focus will be on the rule by which these classical predictions are to be combined to recover the quantum mechanical prediction. Interestingly, this rule is fairly simple, and there is a sense in which it applies uniformly to each component of our imagined experiment, that is, to the preparation, the transformation, and the measurement. We will state this rule shortly. But first we need to describe our discrete, epistemically restricted classical world more precisely.

In our approach, each component of a classical experiment is described at two levels. First we need to specify the "framework"; then we specify a probability distribution within the chosen framework. The framework defines the epistemic restriction that the probability distribution must adhere to. The introduction of the concept of a framework is perhaps the main way in which our formalism differs from Spekkens'.

For the preparation, the framework is a striation of the phase space, which partitions the $d^{2}$ ontic states (that is, the points of phase space) into $d$ parallel lines, each consisting of $d$ points. Within this framework, a classical preparation is represented by a probability dis- tribution over phase space that is required to be uniform over each of the lines of the chosen striation. Thus, as in Spekkens' model, the most that an experimenter can know about the ontic state is that it lies on a particular line. (Note, though, that for Spekkens, there is an allowed epistemic state associated with each line of phase space, whereas for us, once a framework has been chosen, only the lines of a specific striation can play this role.) We use the letter $B$ to label a striation, because, as we explain in Section 2, each striation is associated with an orthogonal basis of the system's Hilbert space.

In our analysis of a transformation (for which we will usually use the word "channel," to avoid potential ambiguities), we begin by considering what we call the basic "ontic transitions." An ontic transition is a transition of the system from a phase-space point $\alpha$ to a phase-space point $\beta$. We label this transition $\alpha \rightarrow \beta$. There are $d^{4}$ ontic transitions. The framework for a channel is labeled by a $2 \times 2$ symplectic matrix $S$ and is a partitioning of the set of ontic transitions into $d^{2}$ "displacement classes," each having $d^{2}$ elements. A displacement class is the set of all ontic transitions $\alpha \rightarrow \beta$ such that $\beta=S \alpha+\delta$, where $\delta$ is a phase-space vector (a displacement) that labels the displacement class within the framework $S$. (Here $\alpha, \beta$, and $\delta$ are all to be thought of a two-component column vectors.) Within this framework, a classical channel is represented by a conditional probability distribution - the probability of $\beta$ given $\alpha$ - that depends only on the displacement class in which the transition $\alpha \rightarrow \beta$ lies. That is, it depends only on the value of the displacement $\delta=\beta-S \alpha$. If we combine this conditional probability distribution with the distribution over $\alpha$ defined by the preparation, it is still the case, after the action of the channel, that the experimenter can know at most what line the ontic state lies on.

Finally we consider the measurement. For the measurement, as for the preparation, the framework is a striation, which we can label $B^{\prime}$. Each outcome $E$ of the measurement is represented by a conditional probability function interpreted as the probability of the outcome $E$ when the system is at the point $\beta$. This function can depend only on which line of $B^{\prime}$ the point $\beta$ lies on.

To summarize, for an experiment consisting of a preparation, a channel, and a measurement, a framework consists of a striation (for the preparation), a symplectic matrix (for the channel), and another striation (for the measurement). We can label the framework by the ordered triple $\left(B^{\prime}, S, B\right)$, where $B$ and $B^{\prime}$ are the striations for the preparation and measurement, respectively. The classical experiment itself is expressed by a set of probability functions, each satisfying an epistemic restriction defined by the framework. The classical theory then yields the conditional probability of the par- 
ticular measurement outcome for the given preparation and channel.

We should note that the "classical theory" consists simply of the ordinary rules of probability. Once we are given an initial probability distribution over the phase space (the preparation), and we are told with what probability the system at any point in phase space will move to any other point (the channel), and with what probability each of these points leads to the outcome $E$, it is straightforward to assign a probability to $E$.

We now come to the question of recovering the standard quantum prediction from the collection of classical predictions. It turns out that this can be done via the following simple rule: to recover the quantum mechanical prediction, we combine the predictions from all the classical experiments by summing their "nonrandom parts," that is, their deviations from what one would have obtained by starting with the uniform distribution over phase space. This sum (possibly divided by a "redundancy factor," as we will explain) is then taken to be the nonrandom part of the quantum prediction, that is, the deviation from what one would have obtained by starting with the completely mixed state. Thus it can easily happen that for each individual classical experiment the final probability distribution is nearly completely random, and yet the quantum prediction is far from random. The reader should understand, by the way, that at this point we do not expect it to be obvious why one should add the nonrandom parts of the classical predictions to get the nonrandom part of the quantum prediction. Showing that this is indeed the case is a large part of what we do in this paper. But this procedure, though quite nonclassical, is at least mathematically plausible: it is related to the familiar tactic of embedding the $d \times d$ density matrices in a $\left(d^{2}-1\right)$ dimensional vector space by subtracting from each matrix the completely mixed state [5].

Though in this paper we begin with a standard quantum description and decompose it into epistemically restricted classical descriptions, we would ultimately like to begin with classical descriptions and show that, under a reasonable set of requirements, they are equivalent to a quantum description. In a certain sense we do this: the global constraints we identify in Appendix D are sufficient to guarantee that a set of classical descriptions will lead to a correct quantum prediction. However, these constraints are more complicated than one would like. We hope they can ultimately be replaced by simpler rules that emerge more naturally from our classical setting. In Section 9 we mention one strategy by which this might be possible.

One might wonder whether our work can be used to construct an ontology of quantum theory, that is, an answer to the question, what is really going on? We do not see any obvious way of doing this, though we do not rule out such an interpretation. One might try to imagine each of our classical observers as having their own, limited perspective on a common reality. Then somehow the quantum world arises from the perspectives of all these classical observers. But their perspectives, expressed in their derived probabilities of the outcomes of a measurement, are combined in a way that differs from any way in which we would normally combine classical probabilities. It is this strange way of combining the classical results (that is, by summing the nonrandom parts) that makes it difficult to extract a picture of anything that could be called "the actual state of affairs."

The above concepts (if not the ontology) will become clearer in Section 4, where we show how summing the nonrandom parts of the classical predictions leads to the quantum prediction. Then, in Section 5, we show that for most of the classical frameworks we have considered, the nonrandom parts are in fact zero, so that those frameworks do not contribute at all to the quantum prediction. We show that the frameworks that might make a nonzero contribution are the ones we call "coherent." This means that the individual components of the framework- $B, S$, and $B^{\prime}$ for an experiment that includes a single channel - are consistent with each other in the sense that the symplectic matrix $S$ takes the original striation $B$ to the final striation $B^{\prime}$. This consistency also makes sense within the classical story we are telling: our imagined classical experimenter would presumably want to analyze the output of the channel in the unique striation that actually reveals the information this output holds.

To prepare the ground for our work in Sections 4 and 5, Section 2 reviews three uses of quasiprobabilities in quantum theory: (i) the discrete Wigner function, which represents a quantum state, (ii) transition quasiprobabilities, which represent a quantum channel, and (iii) the quasiprobability of obtaining a certain measurement outcome when the system is at any given point in phase space. Then in Section 3, we show how these quasiprobabilities can be used to define the actual probability distributions that characterize our classical experiments. Once we have worked out our formalism for prime dimensions and presented an illustrative example in Section 6, we show in Section 7 how to extend our results to any system whose Hilbert-space dimension is a power of an odd prime, such as a set of $n$ qutrits. Powers of 2 and composite dimensions that are not prime powers present special challenges for our formulation; we describe these challenges in Section 8. We draw conclusions in Section 9.

As will become clear in Section 3, our work is closely related to work on quantum state tomography, quan- 
tum process tomography, and quantum measurement tomography. The probability distributions we use to represent preparations, channels and measurement outcomes all arise naturally in tomographic settings.

Our work is also closely related to the "classical" approach to quantum theory pursued in Refs. [6, 7] and other papers by the same authors, in that both approaches use the tomographic representation of quantum states. These authors and their collaborators have built up a large body of work using this kind of representation to describe many quantum phenomena. Although we use a similar technique for the description of states, we treat channels and measurements in a different manner that leads to an evenhanded tomographic description of all aspects of quantum operations and allows a direct comparison to classical operations. Moreover, part of our goal is to create a formulation of quantum mechanics that clearly displays the relationship between an epistemically restricted classical theory and the full quantum theory. The connection between different versions of Spekkens' model and the stabilizer subtheory of quantum mechanics has been thoroughly studied previously $[3,8,9]$

The goal of expressing quantum theory in terms of actual probability distributions has motivated other authors as well. Fuchs and Schack [10] have shown how to express states, transformations, and Born's rule in terms of genuine probability distributions, not over phase space but over the set of outcomes of a symmetric informationally complete measurement. Other researchers have found ways of making transition probabilities nonnegative by adding extra structure to the discrete phase space [11-14]. One recently studied model that does not require negativity reproduces the operational statistics of the $n$-qubit stabilizer formalism [15]. That model is contextual in the traditional sense of Kochen and Specker [16] and Bell [17] and treats the quantum state as epistemic. Zurel et al. go beyond the stabilizer subtheory in introducing a nonnegative probabilistic representation that applies to a specific model of universal quantum computation (quantum computation with magic states) $[18,19]$. Their construction constitutes a hidden-variable model with non-unique representations of states that fits within the standard ontological models framework [20] (it is measurementnoncontextual but preparation-contextual [21]). It thus stands in contrast to our formalism in which every quantum state has a unique representation but which lies beyond the standard ontological models framework because of the unusual rule for combining probabilities. Another difference is that we base our formalism on a standard discrete phase space, whereas Zurel et al. use an expanded phase space.

A word about notation. Probabilities and quasiprob- (a)

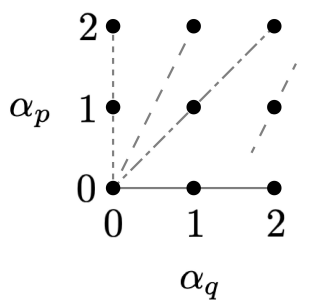

(b)

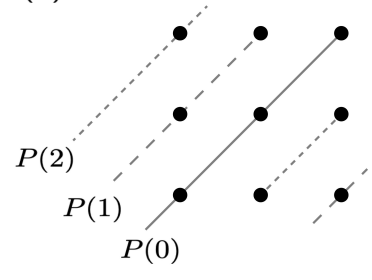

Figure 1: The discrete phase space for a qutrit is pictured as a $3 \times 3$ array of points. Fig. 1(a) shows the four rays-that is, lines passing through the origin-each with different line pattern, that determine striations in the space. In Fig. 1(b), a choice of ray with slope 1 , for example, picks out a striation. Summing the Wigner function over each of the lines of the striation yields a marginal probability distribution. Here each different line pattern identifies a set of three points which all belong to the same phase-space line.

abilities play a few distinct roles in this paper. We represent all quasiprobabilities, including the Wigner function, by the letter $Q$. Probabilities involving ontic states and ontic transitions are labeled $R$, since they are associated with an epistemic restriction. All probabilities associated with genuinely observable events (as opposed to the unobservable ontic states and transitions) are labeled $P$. Finally, we use all of the expressions $Q(\cdot \mid \cdot), R(\cdot \mid \cdot)$, and $P(\cdot \mid \cdot)$ as shorthand symbols for "the probability (or quasiprobability) of [the first argument] given [the second argument]," and not as symbols for fixed mathematical functions. Thus, much of the meaning of the expression comes from the symbols inside the parentheses.

\section{Quasiprobabilities}

Though we aim to express quantum theory in terms of ordinary probability distributions taking only nonnegative values, we begin with a formulation of quantum theory based on quasiprobability distributions. We use these distributions in Section 3 to define our nonnegative probabilities.

\subsection{States}

There are several distinct formulations of discrete phase space and discrete Wigner functions [11-13,22-41]. For the next few sections, we use the formulation proposed in Ref. [24], which is simplest when the dimension $d$ of the system's Hilbert space is a prime number. In Section 7 , where we consider prime-power values of $d$, we use the closely related definition proposed in Refs. [34] and [35]. For the time being, we restrict our attention to the prime-dimensional case. 
The discrete phase space for a system with (prime) dimension $d$ is a two-dimensional vector space over the field $\mathbb{Z}_{d}$, which we picture as a $d \times d$ array of points as discussed in the Introduction. Phase-space points are labeled by Greek letters, and the horizontal and vertical components of a point $\alpha$ are called $\alpha_{q}$ and $\alpha_{p}$, respectively, in reference to the analogy with position and momentum. A line in this phase space is the solution to an equation of the form $a \alpha_{q}+b \alpha_{p}=c$, for $a, b, c \in \mathbb{Z}_{d}$ with $a$ and $b$ not both zero. Two lines satisfying linear equations that differ only in the value of $c$ are called parallel. Fig. 1 shows examples of lines and parallel lines. As we have mentioned in the Introduction, a complete set of parallel lines is called a striation.

A general quantum state with density matrix $w$ is represented in this picture by its Wigner function, defined by

$$
Q(\alpha \mid w)=\frac{1}{d} \operatorname{tr}\left(w A_{\alpha}\right) .
$$

Here the operators $A_{\alpha}$, called "phase-point operators," are a set of orthogonal Hermitian matrices, each with unit trace. For a qubit $(d=2)$, the $A$ 's are defined by

$$
A_{\alpha}=\frac{1}{2}\left[I+(-1)^{\alpha_{p}} X+(-1)^{\alpha_{q}+\alpha_{p}} Y+(-1)^{\alpha_{q}} Z\right],
$$

where $I$ is the $2 \times 2$ identity matrix and $X, Y$, and $Z$ are the Pauli matrices. For odd $d$, the $A$ 's can be defined by their matrix components:

$$
\left(A_{\alpha}\right)_{k l}=\delta_{2 \alpha_{q}, k+l} \omega^{\alpha_{p}(k-l)},
$$

where $\omega=e^{2 \pi i / d}$ and the matrix indices $k$ and $l$ take values in $\mathbb{Z}_{d}$. (So the addition in the subscript of the Kronecker delta is mod $d$.) For both even and odd $d$, the $A$ 's satisfy the equation

$$
\operatorname{tr}\left(A_{\alpha} A_{\beta}\right)=d \delta_{\alpha \beta},
$$

from which it follows that we can invert Eq. (1) to express the density matrix in terms of the Wigner function:

$$
w=\sum_{\alpha} Q(\alpha \mid w) A_{\alpha} .
$$

The Wigner function takes only real values and is normalized like a probability distribution; that is,

$$
\sum_{\alpha} Q(\alpha \mid w)=1
$$

But $Q(\alpha \mid w)$ is not a proper probability distribution as its values can be negative. Nevertheless, the marginal distribution obtained by summing the Wigner function over any set of parallel lines in phase space gives the actual probabilities of the outcomes of an orthogonal measurement. (See Fig. 1(b).) For example, the sums over the vertical lines are the probabilities of the outcomes of a measurement in the standard basis. This property can be traced back to a property of the $A$ operators: for any line $\ell$,

$$
\frac{1}{d} \sum_{\alpha \in \ell} A_{\alpha}=\left|\psi_{\ell}\right\rangle\left\langle\psi_{\ell}\right|
$$

where $\left|\psi_{\ell}\right\rangle$ is a state vector associated with the line $\ell$. Because of the orthogonality of the $A$ operators (Eq. (4)), the state vectors associated with two parallel lines are orthogonal. (We will make use of this fact in Section 3.)

An important set of operators in the discrete phase space picture are the displacement operators $D_{\delta}$. They have the property that

$$
D_{\delta} A_{\alpha} D_{\delta}^{\dagger}=A_{\alpha+\delta} .
$$

For $d=2$, we can write $D_{\delta}$ simply as

$$
D_{\delta}=X^{\delta_{q}} Z^{\delta_{p}} .
$$

For odd $d$, we replace the Pauli matrices $X$ and $Z$ with their natural generalizations, defined in terms of our standard orthonormal basis $\{|m\rangle\}$ by

$$
X|m\rangle=|m+1\rangle \quad Z|m\rangle=\omega^{m}|m\rangle, \quad m \in \mathbb{Z}_{d} .
$$

We also adopt a convenient overall phase factor and write

$$
D_{\delta}=\omega^{\delta_{q} \delta_{p} / 2} X^{\delta_{q}} Z^{\delta_{p}} .
$$

Here the division in the exponent of $\omega$ is understood to be in the field $\mathbb{Z}_{d}$; for example, $\omega^{1 / 2}=\omega^{(d+1) / 2}$.

It is often useful to write the operators $A_{\alpha}$ in terms of the displacement operators. For $d=2$, we have already done this in Eq. (2). For odd $d$, the corresponding expression is

$$
A_{\alpha}=\frac{1}{d} \sum_{\delta} \omega^{\langle\alpha, \delta\rangle} D_{\delta},
$$

where $\langle\cdot, \cdot\rangle$ is the symplectic product:

$$
\langle\alpha, \delta\rangle=\alpha_{p} \delta_{q}-\alpha_{q} \delta_{p} .
$$

Ultimately, it is the relation (12) that gives the set of $A$ 's its special structure (e.g., allowing the above-mentioned interpretation of the marginals).

\subsection{Channels}

In this paper we restrict our attention to transformations of a quantum state that preserve both the dimension of the state space and the normalization of the state. We refer to such transformations as channels. 
(Some authors include the dimension-preserving property in the definition of "channel" as we do, while others allow a channel to change the dimension.) Mathematically, a channel is represented by a completely positive, trace-preserving linear map from the set of operators on a Hilbert space of dimension $d$ to that same set of operators. Such a map can always be described by a set of $d \times d$ Kraus matrices $B_{j}$ satisfying the condition

$$
\sum_{j} B_{j}^{\dagger} B_{j}=I .
$$

In terms of these matrices, the channel can be expressed as

$$
\mathcal{E}(w)=\sum_{j} B_{j} w B_{j}^{\dagger} .
$$

In phase space, a channel $\mathcal{E}$ is fully described by a set of transition quasiprobabilities $Q_{\mathcal{E}}(\beta \mid \alpha)$, from which one can compute the new Wigner function from the old one:

$$
Q(\beta \mid \mathcal{E}(w))=\sum_{\alpha} Q_{\mathcal{E}}(\beta \mid \alpha) Q(\alpha \mid w) .
$$

$\left(Q_{\mathcal{E}}(\beta \mid \alpha)\right.$ is an example of a Liouville representation of a quantum channel [42].) The transition quasiprobabilities representing the channel $\mathcal{E}$ are given by [43-45]

$$
Q_{\mathcal{E}}(\beta \mid \alpha)=\frac{1}{d} \operatorname{tr}\left[A_{\beta} \mathcal{E}\left(A_{\alpha}\right)\right] .
$$

The normalization condition

$$
\sum_{\beta} Q_{\mathcal{E}}(\beta \mid \alpha)=1
$$

is automatically satisfied, but, like the Wigner function, the transition quasiprobabilities can take negative values. Thoughout this paper, we will assume that our channels are unital; that is, they send the completely mixed state to itself. In terms of the transition quasiprobabilities, this means that

$$
\sum_{\alpha} Q_{\mathcal{E}}(\beta \mid \alpha)=1
$$

So summing $Q_{\mathcal{E}}$ over either of its arguments yields unity.

We now come to a fact that we rely heavily on in this paper. There are certain linear transformations acting on the discrete phase space that correspond in a very simple way to unitary transformations acting on Hilbert space [34,46-48]. For such linear transformations, represented by $2 \times 2$ matrices $S$ with entries in $\mathbb{Z}_{d}$, the correspondence is given by

$$
U_{S} A_{\alpha} U_{S}^{\dagger}=A_{S \alpha} \text { for all } \alpha \in \mathbb{Z}_{d}^{2},
$$

where $U_{S}$ is a unitary transformation associated with $S$. That is, $U_{S}$ bears the same relation to the linear transformation $S$ that $D_{\delta}$ bears to the displacement $\delta$. (See Eq. (8).) If such a unitary operator exists for a given $S$, it is unique up to an overall phase factor. (For odd $d$, the uniqueness follows from results in Ref. [47]. For $d=2$, the proof is straightforward.) We will say that a transformation $S$ for which such a unitary transformation exists is a legal linear transformation. For odd $d$, the legal linear transformations are the symplectic transformations [47], that is, the transformations $S$ such that $S^{T} J S=J$, where

$$
J=\left(\begin{array}{cc}
0 & -1 \\
1 & 0
\end{array}\right) .
$$

These are also the unit-determinant transformations. (Note that in a higher-dimensional phase space, not every unit-determinant transformation is symplectic.) For $d=2$, the legal linear transformations are again symplectic, but in that case only three of the six symplectic matrices are legal. The other three are equivalent to antiunitary transformations. The number of legal transformations, for odd $d$, is $d\left(d^{2}-1\right)$, but evidently this formula does not work for $d=2$.

Even for the case of odd $d$, we will sometimes want to restrict our attention to a special set of just $d^{2}-1$ symplectic matrices with the following property: the difference between any two of them has nonzero determinant. (The usefulness of this requirement will become clear in Appendix A.) We call such a special set a "minimal reconstructing set," because it defines the smallest set of classical transformations that can be used to reconstruct the quantum channel. It is known that such a special set exists for dimensions $2,3,5,7$, and 11 [48]. To our knowledge, it is not yet known whether such a set exists for any prime $d$ greater than 11 . (Chau has shown that no set of such matrices forming a group exists for other dimensions [48], but we are not insisting that the minimal reconstructing set form a group.) Indeed, we would be happy to use minimal reconstructing sets exclusively if it were known that such a set exists for every prime $d$. Though the full set of legal matrices works well in our formalism, it entails a certain redundancy that we would avoid if the smaller sets were known to exist. Note that for $d=2$, the complete set of legal symplectic matrices is itself a minimal reconstructing set.

For convenience in the following sections, we define the "redundancy factor" $\mathcal{Z}$ to be the number of symplectic matrices in whatever set we are using (either the set of all legal matrices or a minimal reconstructing set) divided by $d^{2}-1$. Thus, $\mathcal{Z}$ is given by

$\mathcal{Z}= \begin{cases}d & \text { if } d \text { is odd and we are using all legal matrices } \\ 1 & \text { if } d=2, \text { or for any minimal reconstructing set. }\end{cases}$

This notational convention will save us from having to write separate formulas for different cases. Specifically, 
we will use a factor of $1 / \mathcal{Z}$ to compensate for the redundancy associated with using the full set of legal matrices when $d$ is odd.

It is worth writing down an explicit expression for the unitary $U_{S}$ associated with a legal linear transformation $S$. For $d=2$, we label the three legal linear transformations as $\mathcal{I}, \mathcal{R}$, and $\mathcal{L}$, as follows.

$$
\mathcal{I}=\left(\begin{array}{ll}
1 & 0 \\
0 & 1
\end{array}\right) \quad \mathcal{R}=\left(\begin{array}{ll}
0 & 1 \\
1 & 1
\end{array}\right) \quad \mathcal{L}=\left(\begin{array}{ll}
1 & 1 \\
1 & 0
\end{array}\right) .
$$

We have chosen the last two symbols because the transformation $\mathcal{R}$ permutes the nonzero points of phase space by rotating them to the right, that is, clockwise (for our picture of phase space), while $\mathcal{L}$ rotates them to the left. For these matrices, the corresponding unitary transformations are

$$
\begin{array}{ll}
U_{\mathcal{I}} & =I \\
U_{\mathcal{L}} & =\frac{1}{\sqrt{2}}\left(\begin{array}{cc}
1 & -i \\
1 & i
\end{array}\right) .
\end{array}
$$

For odd $d$, let the linear transformation be written as

$$
S=\left(\begin{array}{ll}
v & x \\
y & z
\end{array}\right) .
$$

Then we can write the components of $U_{S}$ as [47]

$$
\left(U_{S}\right)_{k l}= \begin{cases}\frac{1}{\sqrt{d}} \omega^{\frac{1}{2 x}}\left(v l^{2}-2 k l+z k^{2}\right) & \text { if } x \neq 0 \\ \delta_{k, v l} \omega^{\frac{1}{2} v y l^{2}} & \text { if } x=0,\end{cases}
$$

where the indices $k$ and $l$ take the values $0, \ldots, d-1$, and again, the division in the exponent of $\omega$ is to be carried out in the field $\mathbb{Z}_{d}$. It is possible to include additional phase factors in the expressions for $U_{S}$ which guarantee that $U_{S_{1}} U_{S_{2}}=U_{S_{1} S_{2}}[46,47]$. However, in this paper we will not require this property and we have not included those phase factors.

\subsection{Measurements}

A general quantum measurement can be represented by a set of positive-semidefinite operators $E_{k}$ that sum to the identity (a POVM), where $k$ labels the outcome. In this paper we will typically focus on a single outcome, which will we refer to by its POVM operator $E$. This could be any operator satisfying

$$
0 \leq E \leq I,
$$

where the latter inequality simply means that the operator $I-E$ is positive-semidefinite.

When considering how to represent an operator $E$ in phase space, it is useful to start with the Born rule.
Once the representation of the state has been chosen, the Born rule picks out a representation of an event as follows. Calling upon Eq. (5), we have

$$
\operatorname{tr}(E w)=\sum_{\alpha} Q(\alpha \mid w) \operatorname{tr}\left(E A_{\alpha}\right) .
$$

It is then natural to represent $E$ by the function

$$
Q(E \mid \alpha)=\operatorname{tr}\left(E A_{\alpha}\right)
$$

so that the phase-space representation of the Born rule reads as a quasiprobabilistic law of total probability:

$$
P(E \mid w)=\sum_{\alpha} Q(E \mid \alpha) Q(\alpha \mid w) .
$$

The function $Q(E \mid \alpha)$ is then interpreted as the conditional quasiprobability of observing event $E$ when the system is at the phase-space point $\alpha$. Although channels and measurements now both appear as conditional quasiprobability functions, their representations are rather different because of their different roles in the theory. The former is a map between states while the latter is inextricably enmeshed with probabilities of events.

The completely mixed state will play an important part in this work and is represented by the uniform distribution in phase space. The probability of event $E$ occurring when the system is in the completely mixed state can be calculated as

$$
P(E \mid I / d)=\frac{1}{d^{2}} \sum_{\alpha} Q(E \mid \alpha)=\frac{1}{d} \operatorname{tr}(E) .
$$

This is the average value of $Q(E \mid \alpha)$ over all of phase space.

\section{Probabilities from quasiprobabilities}

In this section we show how we convert the quasiprobabilistic functions of the preceding section, which can take negative values, into non-negative functions on phase space whose values can be interpreted as probabilities.

\subsection{States}

\subsubsection{Marginal distributions}

The Wigner function contains complete information about the quantum state $w$. Also containing complete information about the quantum state is the set of marginal distributions, that is, the sums of the Wigner function over the lines of phase space. 
Let $B$ denote any of the $d+1$ striations of phase space. Then the set of values

$$
P^{B}(\ell \mid w)=\sum_{\alpha \in \ell} Q(\alpha \mid w),
$$

for all the lines $\ell$ in the striation $B$, constitutes an actual, non-negative probability distribution. It is the distribution over the possible outcomes of a complete orthogonal measurement associated with $B$ when the system is in the state $w$. That is,

$$
P^{B}(\ell \mid w)=\left\langle\psi_{\ell}|w| \psi_{\ell}\right\rangle,
$$

where the states $\left|\psi_{\ell}\right\rangle$ with $\ell \in B$ constitute an orthogonal basis for the system's Hilbert space, as we discussed in Section 2. Moreover, the bases associated with distinct striations are mutually unbiased; that is, each vector in one basis is an equal-magnitude superposition of the vectors in any of the other bases. (This follows from Eq. (4) and the fact that any two nonparallel lines intersect in exactly one point.)

\subsubsection{Probability distributions over phase space}

In the description of our imagined classical experiment, we will find it useful to have a distribution over the points of phase space, derived from the marginal distributions. Our classical experimenter, when working in the framework characterized by the striation $B$, will assign the probability $R^{B}(\alpha \mid w)$ to the point $\alpha$, where

$$
R^{B}(\alpha \mid w)=\frac{1}{d} P^{B}(\ell \mid w)
$$

for every point $\alpha$ lying on the line $\ell$. That is, the probability of the line $\ell$ is spread uniformly over that line, in keeping with the prohibition against the classical observer having any more detailed knowledge of the ontic state than that it lies on a certain line. Note that though both $Q(\alpha \mid w)$ and $R^{B}(\alpha \mid w)$ are distributions over phase space pertaining to the quantum state $w$, they are quite distinct. The Wigner function is a quasiprobability distribution containing complete information about $w$, whereas $R^{B}(\alpha \mid w)$ is a non-negative probability distribution containing partial information about $w$.

One might wonder why we bother at all with phasespace points, considering that the distribution $R^{B}(\alpha \mid w)$ contains no more information than the distribution $P^{B}(\ell \mid w)$. We do this partly because the phase-space points constitute a set of mutually exclusive possibilities that is shared by all our imagined classical observers. (The $d(d+1)$ lines of phase space, by contrast, do not constitute a set on which it makes sense to define a single probability distribution.) Moreover, since the distributions $R^{B}$ refer to the same sample space as the Wigner function, the conversion formula from the $R^{B}$ 's back to $Q$ is particularly simple, as we now show.

\subsubsection{Reconstructing the Wigner function}

If one has in hand all $d+1$ of the distributions $R^{B}(\alpha \mid w)$, or equivalently, if one has all $d+1$ of the distributions $P^{B}(\ell \mid w)$, one can use them to reconstruct the Wigner function via the following formula:

$$
Q(\alpha \mid w)=\frac{1}{d}\left[\sum_{B} P^{B}(\ell \ni \alpha \mid w)-1\right] .
$$

That is, to find the Wigner function at point $\alpha$, we draw from each marginal distribution the probability of the one line in that striation that contains $\alpha$.

Eq. (35) is a standard equation in quantum state tomography. By some measures, using measurements defined by a complete set of mutually unbiased bases is a particularly efficient way to access the information in a quantum state $[49,50]$. The probabilities obtained from such measurements directly yield the Wigner function via Eq. (35), and the density matrix can then be obtained via Eq. (5).

We can express the content of Eq. (35) somewhat more simply by introducing the concept of the "nonrandom part" of a probability distribution, which we mentioned in the Introduction. We will end up using this concept repeatedly. For any probability or quasiprobability $p$, we define $\Delta p$ to be the difference between the value of $p$ and the value it would have had if the state prepared in the experiment were the completely mixed state (represented by a uniform distribution over phase space) and the channel were the completely randomizing channel, that is, the channel that takes every input state to the completely mixed state. Thus, for the quantities $Q(\alpha \mid w)$ and $P^{B}(\ell \mid w)$ appearing in Eq. (35), we have

$$
\begin{aligned}
\Delta Q(\alpha \mid w) & =Q(\alpha \mid w)-\frac{1}{d^{2}} \\
\Delta P^{B}(\ell \mid w) & =P^{B}(\ell \mid w)-\frac{1}{d} .
\end{aligned}
$$

In terms of the nonrandom parts, Eq. (35) becomes

$$
\Delta Q(\alpha \mid w)=\frac{1}{d} \sum_{B} \Delta P^{B}(\ell \ni \alpha \mid w)=\sum_{B} \Delta R^{B}(\alpha \mid w),
$$

where $\Delta R^{B}(\alpha \mid w)=R^{B}(\alpha \mid w)-1 / d^{2}$, in accordance with our general definition of the nonrandom part. Thus, to get the nonrandom part of the quasiprobability distribution representing a quantum state, we simply sum the nonrandom parts of the corresponding classical distributions $R^{B}$. We will find this form of the equation useful in Section 4. 

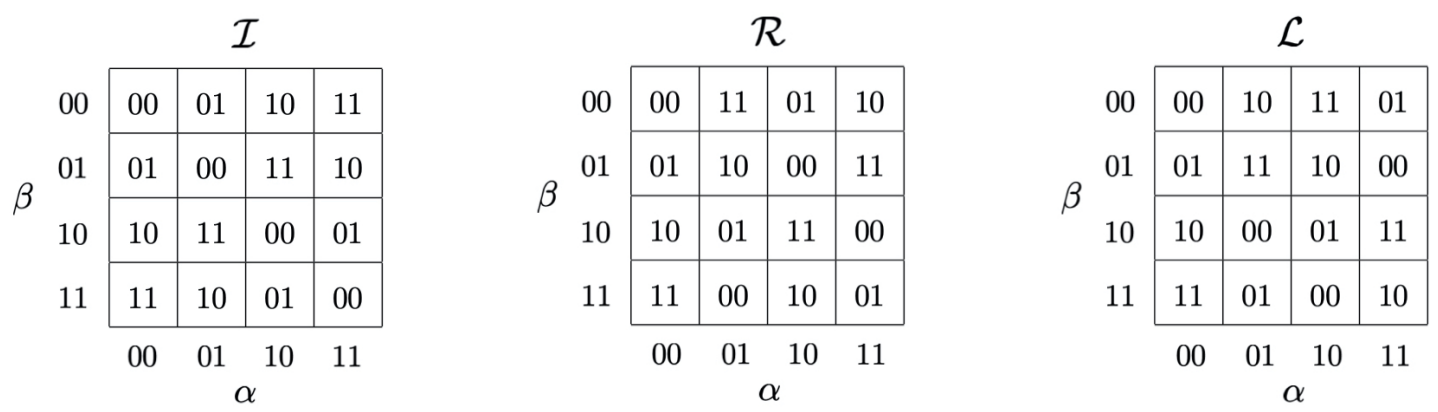

Figure 2: The three partitionings of the ontic transitions $\alpha \rightarrow \beta$ corresponding to the legal symplectic matrices for a qubit. These matrices are $\mathcal{I}, \mathcal{R}$, and $\mathcal{L}$ as given in Eq. (23). The horizontal axis, labeled $\alpha$, indicates the point of origin and the vertical axis, labeled $\beta$, indicates the point of destination. (The designation $\alpha=01$, for example, means that $\alpha_{q}=0$ and $\alpha_{p}=1$.) The entries $00,01,10$, and 11 of the array indicate the displacement that is required to reach $\beta$ after the appropriate symplectic transformation is applied to $\alpha$. Note that any two displacement classes from different grids share exactly one ontic transition.

\subsection{Channels}

\subsubsection{Probabilities of displacement classes}

Our treatment of quantum channels is closely analogous to our treatment of quantum states. A given channel $\mathcal{E}$ is completely characterized by its transition quasiprobabilities $Q_{\mathcal{E}}(\beta \mid \alpha)$. Just as a striation partitions the points of phase space into lines, a symplectic matrix partitions the set of ontic transitions $\alpha \rightarrow \beta$ into displacement classes, as we have discussed in the Introduction. Fig. 2 shows the displacement classes for each of the three legal symplectic matrices for a qubit.

For the symplectic matrix $S$, we define the probability of the displacement class $\delta$ to be

$P_{\mathcal{E}}^{S}(\delta)=\frac{1}{d^{2}} \sum_{\beta, \alpha} \delta_{\beta, S \alpha+\delta} Q_{\mathcal{E}}(\beta \mid \alpha)=\frac{1}{d^{2}} \sum_{\alpha} Q_{\mathcal{E}}(S \alpha+\delta \mid \alpha)$.

That is, we are summing over all the ontic transitions for which the final state $\beta$ is displaced from $S \alpha$ by a specific vector $\delta$. (We can think of the factor $1 / d^{2}$ as a uniform prior for the starting state $\alpha$. It is reasonable to assume this prior when we are describing the channel on its own, as we are doing now. In Subsection 3.2.2 we will define a conditional probability, which, in the context of the whole experiment, will eventually be combined with a distribution over $\alpha$ that need not be uniform.) We now show that, like the marginals discussed in Subsection 3.1.1, the probabilities of the displacement classes are non-negative. We do this by showing how these probabilities arise as the probabilities of observable events in quantum process tomography.

The scenario we have in mind is this. We have an apparatus that effects a quantum channel $\mathcal{E}$. We will apply the apparatus repeatedly, to identically prepared systems, in order to fully characterize the channel. We use an ancilla-assisted method [5,51-55]. That is, the system on which $\mathcal{E}$ will act in each iteration is paired with an ancillary system having the same Hilbert-space dimension $d$. The pair is initially prepared in the simple maximally entangled state

$$
\left|\Psi_{0}\right\rangle=\frac{1}{\sqrt{d}} \sum_{k}|k\rangle \otimes|k\rangle .
$$

Here we are imagining that the system on which the channel $\mathcal{E}$ will act is the second system. We thus apply $\mathcal{E}$ and get the state

$$
\begin{aligned}
w_{\mathcal{E}} & =\sum_{j}\left(I \otimes B_{j}\right)\left|\Psi_{0}\right\rangle\left\langle\Psi_{0}\right|\left(I \otimes B_{j}^{\dagger}\right) \\
& =\frac{1}{d} \sum_{j, k, l}|k\rangle\left\langle l\left|\otimes B_{j}\right| k\right\rangle\langle l| B_{j}^{\dagger}
\end{aligned}
$$

where the $B_{j}$ 's are a set of Kraus operators characterizing the channel $\mathcal{E}$. Now we measure the pair in the orthonormal basis $\left\{\left|\Phi_{\delta}^{S}\right\rangle\right\}$ defined by

$$
\left|\Phi_{\delta}^{S}\right\rangle=\frac{1}{\sqrt{d}} \sum_{m}|m\rangle \otimes D_{\delta} U_{S}|m\rangle
$$

where the index $S$ identifies the basis and the vectors in the basis are labeled by $\delta$. The probability of the outcome $\delta$ is

$$
\begin{aligned}
\left\langle\Phi_{\delta}^{S}\left|w_{\mathcal{E}}\right| \Phi_{\delta}^{S}\right\rangle & =\frac{1}{d^{2}} \sum_{j, k, l}\left\langle k\left|U_{S}^{\dagger} D_{\delta}^{\dagger} B_{j}\right| k\right\rangle\left\langle l\left|B_{j}^{\dagger} D_{\delta} U_{S}\right| l\right\rangle \\
& =\frac{1}{d^{2}} \sum_{j}\left|\operatorname{tr}\left(U_{S}^{\dagger} D_{\delta}^{\dagger} B_{j}\right)\right|^{2} \cdot
\end{aligned}
$$

(That these probabilities are enough to reconstruct the channel $\mathcal{E}$ is shown in Subsection 3.2.3 below.) 
To show that the probability in Eq. (42) is equal to $P_{\mathcal{E}}^{S}(\delta)$, we begin by combining Eqs. (38), (17) and (15):

$$
\begin{aligned}
P_{\mathcal{E}}^{S}(\delta) & =\frac{1}{d^{3}} \sum_{\alpha} \sum_{j} \operatorname{tr}\left(A_{S \alpha+\delta} B_{j} A_{\alpha} B_{j}^{\dagger}\right) \\
& =\frac{1}{d^{3}} \sum_{j} \sum_{\alpha} \operatorname{tr}\left(D_{\delta} U_{S} A_{\alpha} U_{S}^{\dagger} D_{\delta}^{\dagger} B_{j} A_{\alpha} B_{j}^{\dagger}\right) .
\end{aligned}
$$

Now we use the fact that for any matrix $M[45]$,

$$
\sum_{\gamma} A_{\gamma} M A_{\gamma}=d(\operatorname{tr} M) I
$$

This gives us

$$
\begin{aligned}
P_{\mathcal{E}}^{S}(\delta) & =\frac{1}{d^{2}} \sum_{j} \operatorname{tr}\left(U_{S}^{\dagger} D_{\delta}^{\dagger} B_{j}\right) \operatorname{tr}\left(D_{\delta} U_{S} B_{j}^{\dagger}\right) \\
& =\frac{1}{d^{2}} \sum_{j}\left|\operatorname{tr}\left(U_{S}^{\dagger} D_{\delta}^{\dagger} B_{j}\right)\right|^{2},
\end{aligned}
$$

which agrees with the set of probabilities obtained in Eq. (42). Thus, not only is $P_{\mathcal{E}}^{S}(\delta)$ a legitimate probability distribution; it is a distribution one can imagine measuring directly.

Note that in the above derivation, it is crucial that the linear transformation $S$ be equivalent to a unitary transformation, that is, that it be "legal." If we remove this requirement on $S$, it is not hard to find examples where $P_{\mathcal{E}}^{S}(\delta)$ - still defined by Eq. (38) - is negative.

\subsubsection{Conditional distributions over phase space}

For use later when we describe our imagined classical experiment, we now use $P_{\mathcal{E}}^{S}(\delta)$ to define conditional probability distributions over the points of phase space, associated with the channel $\mathcal{E}$. (This is analogous to our defining $R^{B}(\alpha \mid w)$ in Subsection 3.1.2.) We define the probability of the system arriving at $\beta$, given that it starts at $\alpha$, to be

$$
R_{\mathcal{E}}^{S}(\beta \mid \alpha)=P_{\mathcal{E}}^{S}(\beta-S \alpha)
$$

That is, we assume that the probability of the system moving to point $\beta$ when it starts at point $\alpha$ is simply equal to the the probability of the displacement class of the transition $\alpha \rightarrow \beta$ : $R_{\mathcal{E}}^{S}(\beta \mid \alpha)$ does not depend in any other way on the starting point $\alpha$. This is analogous to our assumption, in Subsection 3.1.2, that the probability $R^{B}(\alpha \mid w)$ of a phase-space point $\alpha$ depends only on which line $\alpha$ lies on.

Despite this analogy, the constraint on the form of $R_{\mathcal{E}}^{S}(\beta \mid \alpha)$ is, in a certain respect, less restrictive than the constraint on $R^{B}(\alpha \mid w)$. It allows the observer within the framework $S$ to have complete knowledge of certain deterministic transition rules, namely, those of the form $\alpha \rightarrow S \alpha+\delta$ with a fixed value of $\delta$. The set of all such transformations, from all the frameworks $S$, is the same as the set of reversible transformations allowed in Spekkens' model [1].

We can understand $R_{\mathcal{E}}^{S}(\beta \mid \alpha)$ in another way by writing it in terms of $Q_{\mathcal{E}}(\beta \mid \alpha)$ :

$$
R_{\mathcal{E}}^{S}(\beta \mid \alpha)=\frac{1}{d^{2}} \sum_{\mu} Q_{\mathcal{E}}(S \mu+\delta \mid \mu),
$$

where $\delta=\beta-S \alpha$. Thus, to get the restricted probability distributions $R_{\mathcal{E}}^{S}(\beta \mid \alpha)$, we sum the quasiprobability $Q_{\mathcal{E}}(\beta \mid \alpha)$ over each displacement class associated with $S$ and then spread the result evenly over the $d^{2}$ pairs $(\alpha, \beta)$ in that class, just as, in the case of a preparation, we sum the Wigner function over each line in a striation and then spread the result evenly over that line.

Note that whereas $Q_{\mathcal{E}}(\beta \mid \alpha)$ consists of quasiprobabilities and contains complete information about $\mathcal{E}$, $R_{\mathcal{E}}^{S}(\beta \mid \alpha)$ is non-negative and contains only partial information about $\mathcal{E}$. We now show how, from a knowledge of $R_{\mathcal{E}}^{S}(\beta \mid \alpha)$ for all the legal matrices $S$, or for the matrices in a minimal reconstructing set, one can reconstruct $Q_{\mathcal{E}}(\beta \mid \alpha)$ and thus find $\mathcal{E}$.

\subsubsection{Reconstructing the transition quasiprobabilities}

In this subsection we derive the following formula for reconstructing $Q_{\mathcal{E}}(\beta \mid \alpha)$ from the set of distributions $R_{\mathcal{E}}^{S}(\beta \mid \alpha)$ :

$$
\Delta Q_{\mathcal{E}}(\beta \mid \alpha)=\frac{1}{\mathcal{Z}} \sum_{S} \Delta R_{\mathcal{E}}^{S}(\beta \mid \alpha)
$$

Recall that $\mathcal{Z}$ equals 1 for a qubit or if we are summing over a minimal reconstructing set of symplectic matrices. It equals $d$ if $d$ is odd and we are summing over all the legal symplectics. Here " $\Delta$ " again indicates the nonrandom part. That is,

$$
\begin{aligned}
& \Delta Q_{\mathcal{E}}(\beta \mid \alpha)=Q_{\mathcal{E}}(\beta \mid \alpha)-\frac{1}{d^{2}}, \\
& \Delta R_{\mathcal{E}}^{S}(\beta \mid \alpha)=R_{\mathcal{E}}^{S}(\beta \mid \alpha)-\frac{1}{d^{2}} .
\end{aligned}
$$

So one needs to add the "random part" $1 / d^{2}$ to the left-hand side of Eq. (48) to recover $Q$ itself.

To derive Eq. (48), we begin by rewriting Eq. (38) in terms of the nonrandom parts:

$$
\Delta P_{\mathcal{E}}^{S}(\delta)=\frac{1}{d^{2}} \sum_{\beta, \alpha} \delta_{\beta, S \alpha+\delta} \Delta Q_{\mathcal{E}}(\beta \mid \alpha) .
$$

It is helpful to insert another Kronecker delta and a 
factor of $1 / \mathcal{Z}$ :

$$
\begin{aligned}
\frac{1}{\mathcal{Z}} \sum_{S, \delta} & \delta_{\beta, S \alpha+\delta} \Delta P_{\mathcal{E}}^{S}(\delta) \\
& =\frac{1}{\mathcal{Z} d^{2}} \sum_{S, \delta, \beta^{\prime}, \alpha^{\prime}} \delta_{\beta, S \alpha+\delta} \delta_{\beta^{\prime}, S \alpha^{\prime}+\delta} \Delta Q_{\mathcal{E}}\left(\beta^{\prime} \mid \alpha^{\prime}\right) .
\end{aligned}
$$

Now we use a fact proved in Appendix A:

$$
\frac{1}{\mathcal{Z} d^{2}} \sum_{S, \delta} \delta_{\beta, S \alpha+\delta} \delta_{\beta^{\prime}, S \alpha^{\prime}+\delta}=\delta_{\beta \beta^{\prime}} \delta_{\alpha \alpha^{\prime}}+\frac{1}{d^{2}}\left(1-\delta_{\beta \beta^{\prime}}-\delta_{\alpha \alpha^{\prime}}\right) \text {. }
$$

When we insert this equation into the right-hand side of Eq. (51), most of the terms yield zero when summed over $\beta^{\prime}$ and $\alpha^{\prime}$. This is because $\sum_{\beta^{\prime}} \Delta Q_{\mathcal{E}}\left(\beta^{\prime} \mid \alpha^{\prime}\right)=0$ (since $Q_{\mathcal{E}}$ is a normalized distribution) and $\sum_{\alpha^{\prime}} \Delta Q_{\mathcal{E}}\left(\beta^{\prime} \mid \alpha^{\prime}\right)=0$ (since the channel is unital). We are left with

$$
\frac{1}{\mathcal{Z}} \sum_{S, \delta} \delta_{\beta, S \alpha+\delta} \Delta P_{\mathcal{E}}^{S}(\delta)=\Delta Q_{\mathcal{E}}(\beta \mid \alpha) .
$$

We can rewrite this relation as

$$
\Delta Q_{\mathcal{E}}(\beta \mid \alpha)=\frac{1}{\mathcal{Z}} \sum_{S} \Delta P_{\mathcal{E}}^{S}(\beta-S \alpha)=\frac{1}{\mathcal{Z}} \sum_{S} \Delta R_{\mathcal{E}}^{S}(\beta \mid \alpha),
$$

which is Eq. (48). Thus, summing the nonrandom parts of the $R^{S}$ 's of the restricted classical theory - and if necessary, dividing by the redundancy factor-yields the nonrandom part of the quantum quasiprobability distribution $Q_{\mathcal{E}}$.

\subsection{Measurements}

\subsubsection{Probability conditioned on line states}

Again, a measurement outcome $E$ is represented in phase space by the function

$$
Q(E \mid \alpha)=\operatorname{tr}\left(E A_{\alpha}\right) .
$$

Though this function does not have the normalization of a quasiprobability distribution, it will still be convenient to use our " $\Delta$ " notation with $Q(E \mid \alpha)$ to indicate that we must subtract the probability of the event $E$ given that the measured state is the maximally mixed state (cf. Eq. 31). That is, we define

$$
\Delta Q(E \mid \alpha)=Q(E \mid \alpha)-\frac{1}{d} \operatorname{tr} E .
$$

Or, purely in terms of phase-space concepts,

$$
\Delta Q(E \mid \alpha)=Q(E \mid \alpha)-\frac{1}{d^{2}} \sum_{\mu} Q(E \mid \mu) .
$$

Note that the sum of $\Delta Q(E \mid \alpha)$ over $\alpha$ is zero, just as the sum of $\Delta Q(\alpha \mid w)$ is zero for a density matrix $w$.

According to Eq. (30), the probability $P(E \mid \ell)$ of getting outcome $E$ when the system is in the pure state $\left|\psi_{\ell}\right\rangle$ associated with the line $\ell$ is given by

$$
\begin{aligned}
P(E \mid \ell) & \equiv\left\langle\psi_{\ell}|E| \psi_{\ell}\right\rangle \\
& =\sum_{\alpha} Q(E \mid \alpha) Q\left(\alpha \mid \psi_{\ell}\right) \\
& =\frac{1}{d} \sum_{\alpha \in \ell} Q(E \mid \alpha) .
\end{aligned}
$$

This quantity is of course always non-negative, even though $Q(E \mid \alpha)$ can be negative.

\subsubsection{Probability conditioned on a phase-space point}

Suppose our classical experimenter is using the striation $B$ to analyze the measurement. For such an experimenter, the outcome $E$ will be described by a probability function $R^{B}(E \mid \alpha)$, interpreted as the probability of the outcome $E$ when the system is at the phase-space point $\alpha$. In the framework $B$, all that can matter about the point $\alpha$ is which line it is on in the striation $B$. So we define

$$
R^{B}(E \mid \alpha)=P^{B}(E \mid \ell \ni \alpha),
$$

where $P^{B}(E \mid \ell)$ is the $P(E \mid \ell)$ of Eq. (58) but with the understanding that $\ell$ is chosen from the striation $B$. Thus $R^{B}(E \mid \alpha)$ is the average of $Q(E \mid \beta)$ over all the points $\beta$ lying on the line in $B$ that contains $\alpha$.

\subsubsection{Recovering $Q(E \mid \alpha)$ from the $R^{B \text { 's }}$}

We now show how to recover the function $Q(E \mid \alpha)$ from a knowledge of all the $R^{B}$ 's. This is analogous to recovering the Wigner function from its marginals, but the situation is somewhat different in that $R^{B}(E \mid \alpha)$ is a probability of $E$ rather than a probability of $\alpha$.

To begin, for any fixed $\alpha$ let us consider the sum

$$
\sum_{B} P^{B}(E \mid \ell \ni \alpha)
$$

Each term in this sum is itself an average of $d$ values of $Q(E \mid \beta)$, namely, the ones with $\beta \in \ell$. When we sum over all the lines passing through $\alpha$, the term $Q(E \mid \alpha)$ appears $d+1$ times, once for each of the $d+1$ lines passing through $\alpha$. And any term $Q(E \mid \beta)$ with $\beta \neq \alpha$ appears exactly once, since only one line passes through both $\alpha$ and $\beta$. Thus we have

$$
\begin{aligned}
\sum_{B} P^{B}(E \mid \ell \ni \alpha) & =\frac{1}{d}\left(d Q(E \mid \alpha)+\sum_{\beta} Q(E \mid \beta)\right)(61) \\
& =Q(E \mid \alpha)+\operatorname{tr} E .
\end{aligned}
$$


Solving for $Q(E \mid \alpha)$, we get

$$
Q(E \mid \alpha)=\sum_{B} P^{B}(E \mid \ell \ni \alpha)-\operatorname{tr} E .
$$

As in Section 3.1, the content of this equation looks a bit simpler if we express it in terms of the nonrandom parts. We have defined $\Delta Q(E \mid \alpha)$ in Eq. (56) and according to our general rule, we have

$$
\begin{aligned}
& \Delta P^{B}(E \mid \ell \ni \alpha)=P^{B}(E \mid \ell \ni \alpha)-\frac{1}{d} \operatorname{tr} E ; \\
& \Delta R^{B}(E \mid \alpha)=R^{B}(E \mid \alpha)-\frac{1}{d} \operatorname{tr} E .
\end{aligned}
$$

Inserting these definitions into Eq. (63), we get

$$
\Delta Q(E \mid \alpha)=\sum_{B} \Delta P^{B}(E \mid \ell \ni \alpha)=\sum_{B} \Delta R^{B}(E \mid \alpha) .
$$

One obtains $Q(E \mid \alpha)$ by adding to the left-hand side of this equation the random part $(1 / d) \operatorname{tr} E$. Note that the form of Eq. (65) matches that of Eq. (37).

Eq. (65) provides a way of doing measurement tomography [52], just as some of our earlier equations are associated with state and process tomography. If a measuring device yields an outcome whose mathematical description is unknown, one can send into the device multiple copies of systems prepared in the pure states associated with the lines $\ell$ of phase space. By estimating $P(E \mid \ell)$, one can use Eq. (65) to determine the function $Q(E \mid \alpha)$, which completely characterizes the measurement outcome.

\section{The whole experiment: summing the nonrandom parts}

We now have the necessary tools to express quantum probabilities for a whole experiment in terms of restricted classical probabilities, for systems with primedimensional Hilbert spaces. The simplest quantum experiment is the preparation and subsequent measurement of a quantum state. For this scenario, we will explicitly show how a collection of classical experiments with our epistemic restriction can be combined to give a probabilistic prediction equivalent to the Born rule. We will then broaden our scope to include intervening channels.

The usual quantum mechanical description of a prepare-and-measure experiment entails the specification of a state as a density matrix $w$ and a measurement outcome as a POVM element $E$. The Born rule can be stated using the standard operators on a Hilbert space or with quasiprobabilistic quantities (Eq. (30)):

$$
P(E \mid w) \equiv \operatorname{tr}[E w]=\sum_{\alpha} Q(E \mid \alpha) Q(\alpha \mid w) .
$$

Through the relationships developed in Section 3, the Wigner function description acts as a stepping stone towards yet another equivalent representation of the content of the Born rule.

The first goal of the present section is to explain how the content of the Born rule can be reproduced, in terms of nonrandom parts, as

$$
\Delta P(E \mid w)=\sum_{\mathcal{F}} \Delta R^{\mathcal{F}}(E \mid w),
$$

where $\mathcal{F}$ is shorthand for the framework $\left(B^{\prime}, B\right)$ of the whole experiment, and the classical probability $R^{\mathcal{F}}(E \mid w)$ is defined by

$$
R^{\mathcal{F}}(E \mid w)=\sum_{\alpha} R^{B^{\prime}}(E \mid \alpha) R^{B}(\alpha \mid w) .
$$

That is, $R^{\mathcal{F}}$ reflects the completely classical probabilistic reasoning that would be implemented by a classical experimenter subject to an epistemic restriction. The claim, then, is that the nonrandom part of the quantum prediction is equal to the sum of the nonrandom parts of classical predictions given by all the possible classical frameworks for preparations and measurements.

Before we prove Eq. (67), let us give it a more physical meaning by interpreting $R^{B}(\alpha \mid w)$ and $R^{B^{\prime}}(E \mid \alpha)$ in terms of loss of quantum coherence. In going from $Q(\alpha \mid w)$ to $R^{B}(\alpha \mid w)$, we have, in effect, implemented a dephasing of the state $w$ in the basis $B$. Similarly, we can get $R^{B^{\prime}}(E \mid \alpha)$ from $Q(E \mid \alpha)$ by imagining a dephasing in the basis $B^{\prime}$ prior to the measurement. If $B^{\prime}$ does not match $B$, the expression given in Eq. (68) contributes nothing to the nonrandom part in Eq. (67). (This claim is justified and generalized in Section 5.) So the important terms in Eq. (67) are those with $B=B^{\prime}$. Each of these terms corresponds to a world that is classical in the sense that the quantum coherence in the basis $B$ has been eliminated. Eq. (67) tells us how the predictions of the classical experiments for all the bases $B$ are to be combined to produce the quantum prediction.

We now prove Eq. (67). Starting with the right-hand side of the equation, or "RHS ${ }^{E, w}$," we insert the definition (68) and distribute the $\Delta$ into the phase-space summation as described in Appendix B:

$$
\begin{aligned}
\operatorname{RHS}^{E, w} & =\sum_{B, B^{\prime}} \Delta\left[\sum_{\alpha} R^{B^{\prime}}(E \mid \alpha) R^{B}(\alpha \mid w)\right] \\
& =\sum_{B, B^{\prime}}\left[\sum_{\alpha} \Delta R^{B^{\prime}}(E \mid \alpha) \Delta R^{B}(\alpha \mid w)\right] .
\end{aligned}
$$

Performing the summations over the classical frameworks used for the preparations and measurements and 
recalling Eqs. (37) and (65), we move to an expression involving only quasiprobabilities, finding

$$
\begin{aligned}
\operatorname{RHS}^{E, w} & =\sum_{\alpha} \Delta Q(E \mid \alpha) \Delta Q(\alpha \mid w) \\
& =\sum_{\alpha}\left(Q(E \mid \alpha)-\frac{1}{d} \operatorname{tr}(E)\right)\left(Q(\alpha \mid w)-\frac{1}{d^{2}}\right) .
\end{aligned}
$$

Doing the sum and using Eqs. (6) and (31), we have a cancellation of certain constant terms, leaving the desired result,

$$
\mathrm{RHS}^{E, w}=P(E \mid w)-\frac{1}{d} \operatorname{tr}(E)=\Delta P(E \mid w) .
$$

It is not much more work to include a channel. In complete analogy with our result for the prepare-andmeasure experiment, we will show that

$$
\Delta P(E \mid \mathcal{E}, w)=\frac{1}{\mathcal{Z}} \sum_{\mathcal{F}} \Delta R^{\mathcal{F}}(E \mid \mathcal{E}, w)
$$

where $\mathcal{F}$ is now the whole framework $\mathcal{F}=\left(B^{\prime}, S, B\right)$ and $R^{\mathcal{F}}(E \mid \mathcal{E}, w)$ is defined to be

$$
R^{\mathcal{F}}(E \mid \mathcal{E}, w)=\sum_{\beta, \alpha} R^{B^{\prime}}(E \mid \beta) R_{\mathcal{E}}^{S}(\beta \mid \alpha) R^{B}(\alpha \mid w) .
$$

Let us refer to the right-hand side of Eq. (72) as "RHS ${ }^{E, \mathcal{E}, w}$." Again, Appendix B allows for distribution of the $\Delta$ inside the phase-space sum. The relationships in Eqs. (37), (48), (65) lead to

$$
\operatorname{RHS}^{E, \mathcal{E}, w}=\sum_{\beta, \alpha} \Delta Q(E \mid \beta) \Delta Q_{\mathcal{E}}(\beta \mid \alpha) \Delta Q(\alpha \mid w) .(74)
$$

Unwrapping the $\Delta$ terms as a difference between the $Q$ 's and the random parts, we find that there are eight terms after multiplication. One of them contains three $Q$ factors. The other seven terms can be simplified using the following equations:

$$
\begin{aligned}
& \sum_{\alpha} Q(\alpha \mid w)=1 \\
& \sum_{\alpha} Q_{\mathcal{E}}(\beta \mid \alpha)=\sum_{\beta} Q_{\mathcal{E}}(\beta \mid \alpha)=1 \\
& \sum_{\beta} Q(E \mid \beta)=d \operatorname{tr}(E) .
\end{aligned}
$$

The key thing to notice is that if there is an unpaired phase-space point among factors of $Q$ 's, the sum over that point can be performed using one of the preceding equations. This will leave at least one other point unpaired and in a cascading fashion, the term can be reduced to a constant of magnitude $\frac{1}{d} \operatorname{tr}(E)$. The sign of the constant term is determined by how many factors of $-\frac{1}{d^{2}}$ or $-\frac{1}{d} \operatorname{tr}(E)$ went into it. There are three positive terms and four negative terms, leaving us with

$$
\begin{aligned}
\operatorname{RHS}^{E, \mathcal{E}, w} & =\sum_{\beta, \alpha} Q(E \mid \beta) Q_{\mathcal{E}}(\beta \mid \alpha) Q(\alpha \mid w)-\frac{1}{d} \operatorname{tr}(E) \\
& =\Delta P(E \mid \mathcal{E}, w)
\end{aligned}
$$

which is what we wished to show.

There needs to be very little tweaking of the preceding argument to generalize to any number of intervening channels between preparation and measurement. We will find that if $n$ channels are implemented, with $\mathcal{E}_{1}$ acting first and $\mathcal{E}_{n}$ acting last, the probability of the outcome $E$ is determined by

$$
\Delta P\left(E \mid \mathcal{E}_{n}, \ldots, \mathcal{E}_{1}, w\right)=\frac{1}{\mathcal{Z}^{n}} \sum_{\mathcal{F}} \Delta R^{\mathcal{F}}\left(E \mid \mathcal{E}_{n}, \ldots, \mathcal{E}_{1}, w\right),
$$

where $\mathcal{F}=\left(B^{\prime}, S_{n}, \ldots, S_{1}, B\right)$ and

$$
R^{\mathcal{F}}\left(E \mid \mathcal{E}_{n}, \ldots, \mathcal{E}_{1}, w\right)=\sum_{\beta_{n}, \ldots, \beta_{1}, \alpha} R^{B^{\prime}}\left(E \mid \beta_{n}\right) R_{\mathcal{E}_{n}}^{S_{n}}\left(\beta_{n} \mid \beta_{n-1}\right) \cdots R_{\mathcal{E}_{1}}^{S_{1}}\left(\beta_{1} \mid \alpha\right) R^{B}(\alpha \mid w) .
$$

The only point requiring a new argument comes at the step that starts with an equation analogous to Eq. (74):

$$
\operatorname{RHS}^{E, \mathcal{E}_{n}, \ldots, \mathcal{E}_{1}, w}=\sum_{\beta_{n}, \ldots, \beta_{1}, \alpha} \Delta Q\left(E \mid \beta_{n}\right) \Delta Q_{\mathcal{E}_{n}}\left(\beta_{n} \mid \beta_{n-1}\right) \cdots \Delta Q_{\mathcal{E}_{1}}\left(\beta_{1} \mid \alpha\right) \Delta Q(\alpha \mid w) .
$$

Unwrapping the $\Delta$ 's and multiplying out all the factors gives $2^{n+2}$ terms. One of those is not a constant and just contains factors of $Q$ 's. Again, because of a cascading simplification as the sum over phase-space points is performed, the other terms will all be constants of magnitude $\frac{1}{d} \operatorname{tr}(E)$. The key is to count the number of terms with each sign and compare those numbers.

Let $k$ be the number of constant factors, i.e. $-\frac{1}{d^{2}}$ or $-\frac{1}{d} \operatorname{tr}(E)$, that go into such a term. If $k$ is even, the term will be positive while if $k$ is odd, the term will be negative. There will be $n+2$ choose $k$ terms having $k$ constant 
factors, because we have $n+2$ factors of $\Delta Q$ in Eq. (81). This means we have

$$
\operatorname{RHS}^{E, \mathcal{E}_{n}, \ldots, \mathcal{E}_{1}, w}=\left(\sum_{\beta_{n}, \beta_{n-1}, \ldots, \beta_{1}, \alpha} Q\left(E \mid \beta_{n}\right) Q_{\mathcal{E}_{n}}\left(\beta_{n} \mid \beta_{n-1}\right) \cdots Q_{\mathcal{E}_{1}}\left(\beta_{1} \mid \alpha\right) Q(\alpha \mid w)\right)+\frac{1}{d} \operatorname{tr}(E) \sum_{k=1}^{n+2}(-1)^{k}\left(\begin{array}{c}
n+2 \\
k
\end{array}\right) .
$$

We now use an identity that follows from the binomial theorem:

$$
\sum_{k=1}^{m}(-1)^{k}\left(\begin{array}{c}
m \\
k
\end{array}\right)=\sum_{k=0}^{m}(-1)^{k}\left(\begin{array}{c}
m \\
k
\end{array}\right)-1=-1,
$$

which leads us directly to Eq (79).

\section{Coherent frameworks}

For an experiment consisting of a preparation, a channel, and a measurement, we showed in the preceding section that

$$
\Delta P(E \mid \mathcal{E}, w)=\frac{1}{\mathcal{Z}} \sum_{\mathcal{F}} \Delta R^{\mathcal{F}}(E \mid \mathcal{E}, w)
$$

where

$$
\Delta R^{\mathcal{F}}(E \mid \mathcal{E}, w)=\Delta\left[\sum_{\beta, \alpha} R^{B^{\prime}}(E \mid \beta) R_{\mathcal{E}}^{S}(\beta \mid \alpha) R^{B}(\alpha \mid w)\right]
$$

(Again, $\mathcal{F}=\left(B^{\prime}, S, B\right)$.) We now show that unless $B^{\prime}$ is equal to $S B$, the expression in Eq. (85) is zero and thus does not contribute to the quantum probability $P(E \mid \mathcal{E}, w)$. That is, the only frameworks for the whole experiment that contribute to the probability of the outcome $E$ are the coherent frameworks - those for which the symplectic transformation $S$ takes the lines of $B$ to the lines of $B^{\prime}$.

We begin by rewriting Eq. (85) by distributing the $\Delta$ as we have done before:

$$
\begin{aligned}
\Delta R^{\mathcal{F}}(E \mid \mathcal{E}, w) & =\sum_{\beta, \alpha} \Delta R^{B^{\prime}}(E \mid \beta) \Delta R_{\mathcal{E}}^{S}(\beta \mid \alpha) \Delta R^{B}(\alpha \mid w) . \\
& =\sum_{\beta} \Delta R^{B^{\prime}}(E \mid \beta) f^{S B}(\beta),
\end{aligned}
$$

where

$$
f^{S B}(\beta) \equiv \sum_{\alpha} \Delta R_{\mathcal{E}}^{S}(\beta \mid \alpha) \Delta R^{B}(\alpha \mid w)
$$

We now wish to show that $f^{S B}(\beta)$ is uniform over any line in the striation $S B$.

To do this, we rewrite $f^{S B}$ using the definition of $R^{S}$ :

$$
f^{S B}(\beta)=\sum_{\alpha} \Delta P_{\mathcal{E}}^{S}(\beta-S \alpha) \Delta R^{B}(\alpha \mid w) .
$$

We want to show that the quantity in Eq. (88) does not change when we replace $\beta$ with a point $\beta^{\prime}$ lying on the same line as $\beta$ in the striation $S B$. Let $\beta^{\prime}$ be such a point, and let $\delta=\beta^{\prime}-\beta$. Then we can write

$$
f^{S B}\left(\beta^{\prime}\right)=\sum_{\alpha} \Delta P_{\mathcal{E}}^{S}\left(\beta-S\left(\alpha-S^{-1} \delta\right)\right) \Delta R^{B}(\alpha \mid w) .
$$

Now let $\alpha^{\prime}=\alpha-S^{-1} \delta$. Then $\alpha$ and $\alpha^{\prime}$ lie on the same line in the striation $B$. Therefore, since $R^{B}(\alpha \mid w)$ is uniform over each such line, we have

$$
\begin{aligned}
f^{S B}\left(\beta^{\prime}\right) & =\sum_{\alpha^{\prime}} \Delta P_{\mathcal{E}}^{S}\left(\beta-S \alpha^{\prime}\right) \Delta R^{B}\left(\alpha^{\prime} \mid w\right) \\
& =f^{S B}(\beta) .
\end{aligned}
$$

So $f^{S B}$ is indeed uniform over any line in $S B$.

We now return to the equation

$$
\Delta P(E \mid \mathcal{E}, w)=\frac{1}{\mathcal{Z}} \sum_{B^{\prime}, S, B} \sum_{\beta} \Delta R^{B^{\prime}}(E \mid \beta) f^{S B}(\beta) .
$$

Holding $B^{\prime}, S$ and $B$ fixed for now, let us imagine what is happening when we sum over $\beta$ if $B^{\prime}$ is not the same as $S B$. Each value of $\beta$ picks out a unique line $\ell^{\prime}$ in the striation $B^{\prime}$ and a unique line $\ell$ in the striation $S B$. As we sum over $\beta$, we are in fact summing over all possible pairs $\left(\ell^{\prime}, \ell\right)$. So we can rewrite Eq. (91) as

$$
\Delta P(E \mid \mathcal{E}, w)=\frac{1}{\mathcal{Z}} \sum_{B^{\prime}, S, B} \sum_{\ell^{\prime}, \ell} \Delta P^{B^{\prime}}\left(E \mid \ell^{\prime}\right) g^{S B}(\ell),
$$

where $g^{S B}(\ell)=f^{S B}(\alpha \in \ell)$. The sums over $\ell^{\prime}$ and $\ell$ are now independent of each other, and the first of these sums has the value zero because of the way $\Delta$ is defined. So any triple $\left(B^{\prime}, S, B\right)$ for which $B^{\prime}$ is different from $S B$ does not contribute to $P(E \mid \mathcal{E}, w)$.

An iterated version of this argument works when there is a sequence of channels $\mathcal{E}_{n}, \ldots, \mathcal{E}_{1}$ : for a framework $\left(B^{\prime}, S_{n}, \ldots, S_{1}, B\right)$ to contribute, we must have $B^{\prime}=S_{n} \cdots S_{1} B$.

As we noted in the Introduction, it makes sense that our imagined classical experimenter, in choosing a framework, would want to choose a framework for the measurement that "matched" the framework for the rest of the experiment. Any striation other than $B^{\prime}=S_{n} \ldots S_{1} B$ would make for a measurement incapable of revealing any information about what had 
gone before. Indeed, we could have insisted from the beginning that what we mean by a framework for the experiment is a coherent framework. We have shown in this section that this approach yields exactly the same result as one obtains with the much larger sum, over all values of $B^{\prime}, S_{n}, \ldots, S_{1}, B$ and with no constraint on the relation among these elements. The larger sum simply includes many terms that are equal to zero.

\section{An example}

As a simple example, consider a photon that has passed through the first beamsplitter of a Mach-Zehnder interferometer and is about to pass through the second beamsplitter before being detected. States of light in the two possible paths are labeled $|0\rangle$ and $|1\rangle$. The beamsplitters are unbalanced such that each has a probability $3 / 4$ of direct transmission (which preserves the value of $j$ in $|j\rangle$ ) and $1 / 4$ of reflection (which flips $j$ ). Specifically, let us take the unitary transformation representing the beamsplitter to be

$$
U=\frac{\sqrt{3}}{2} I+\frac{i}{2} X .
$$

The state prepared by the first beamsplitter is $w=$ $\frac{\sqrt{3}}{2}|0\rangle+\frac{i}{2}|1\rangle$. The Born rule gives a probability of $1 / 4$ that the photon will be found in the path $|0\rangle$ after the second beamsplitter.

This section contains a careful walk-through of how the collection of classical experiments we have described above will reproduce this prediction. Fig. 3 shows how one might visualize the steps leading from quasiprobabilities to classical probabilities and then finally to a prediction in agreement with the Born rule. All these steps in the figure are described in what follows.

This example allows us to display the mechanics of constructing the classical entities from the quantum ones. One can see how quasiprobabilities with distinctly quantum negative values are rendered classical as positive probability distributions at each step of the process. Negativity then returns as we sum the nonrandom parts but this is always made positive by adding in the uniform distribution at the end.

The initial superposition state has a discrete Wigner function

$$
Q(\alpha \mid w)=\frac{1}{8} \begin{array}{|l|l|}
\hline 3-\sqrt{3} & 1+\sqrt{3} \\
\hline 3+\sqrt{3} & 1-\sqrt{3} \\
\hline
\end{array}
$$

Note the sign of the value at the phase space point 10 (read as "(1,0)" - bottom right). We proceed towards the classical representation by calculating the marginal probabilities over the horizontal, diagonal, and vertical striations that we label with $X, Y$, and $Z$, respectively. The two lines in each striation will be labeled with $x=0$ and $x=1$, where all lines that intersect the origin (bottom left) are labeled 0 . The marginal probability distributions for the different striations are

$$
\begin{aligned}
& P^{X}(x)= \begin{cases}\frac{1}{2} & \text { if } x=0 \\
\frac{1}{2} & \text { if } x=1,\end{cases} \\
& P^{Y}(x)= \begin{cases}\frac{1}{4}(2+\sqrt{3}) & \text { if } x=0 \\
\frac{1}{4}(2-\sqrt{3}) & \text { if } x=1,\end{cases} \\
& P^{Z}(x)= \begin{cases}\frac{3}{4} & \text { if } x=0 \\
\frac{1}{4} & \text { if } x=1,\end{cases}
\end{aligned}
$$

Any single classical experimenter may have knowledge of at most one of these distributions. With each $P$ value spread uniformly over its line, the epistemic state of knowledge given in Eq. (34) will be one of the following:

$$
\begin{aligned}
& R^{X}(\alpha \mid w)=\frac{1}{4} \begin{array}{|l|l|}
\hline 1 & 1 \\
\hline 1 & 1 \\
\hline
\end{array} \\
& R^{Y}(\alpha \mid w)=\frac{1}{8} \begin{array}{|l|l|}
\cline { 2 - 3 } 2-\sqrt{3} & 2+\sqrt{3} \\
\cline { 2 - 3 } 2+\sqrt{3} & 2-\sqrt{3} \\
\hline
\end{array} \\
& R^{Z}(\alpha \mid w)=\frac{1}{8} \begin{array}{|l|l|}
\hline 3 & 1 \\
\hline 3 & 1 \\
\hline
\end{array}
\end{aligned}
$$

The classical transformations allowed in the experiments are all stochastic combinations of permutations (the affine symplectic transformations) so the uniform distribution will always be mapped to itself. We are only interested in the nonrandom parts of distributions so $R^{X}$ will have no effect on the calculation going forward and can be ignored.

Moving on to the classical transformations associated with the unitary operation specified above, we start with the transition quasiprobabilities that contain full information about the quantum channel. Employing Eq. (17), and treating $Q_{U}(\beta \mid \alpha)$ as a matrix with $\beta$ labeling the rows and $\alpha$ labeling the columns (both ordered $00,01,10,11$ ), we have

$$
Q_{U}=\frac{1}{4}\left(\begin{array}{cccc}
1 & \sqrt{3} & 3 & -\sqrt{3} \\
-\sqrt{3} & 1 & \sqrt{3} & 3 \\
3 & -\sqrt{3} & 1 & \sqrt{3} \\
\sqrt{3} & 3 & -\sqrt{3} & 1
\end{array}\right) .
$$

From here, the allowed ontic transitions in a specific classical framework can be calculated using Eq. (46) where we treat $R_{\mathcal{E}}^{S}(\beta \mid \alpha)$ as a matrix with rows labelled 


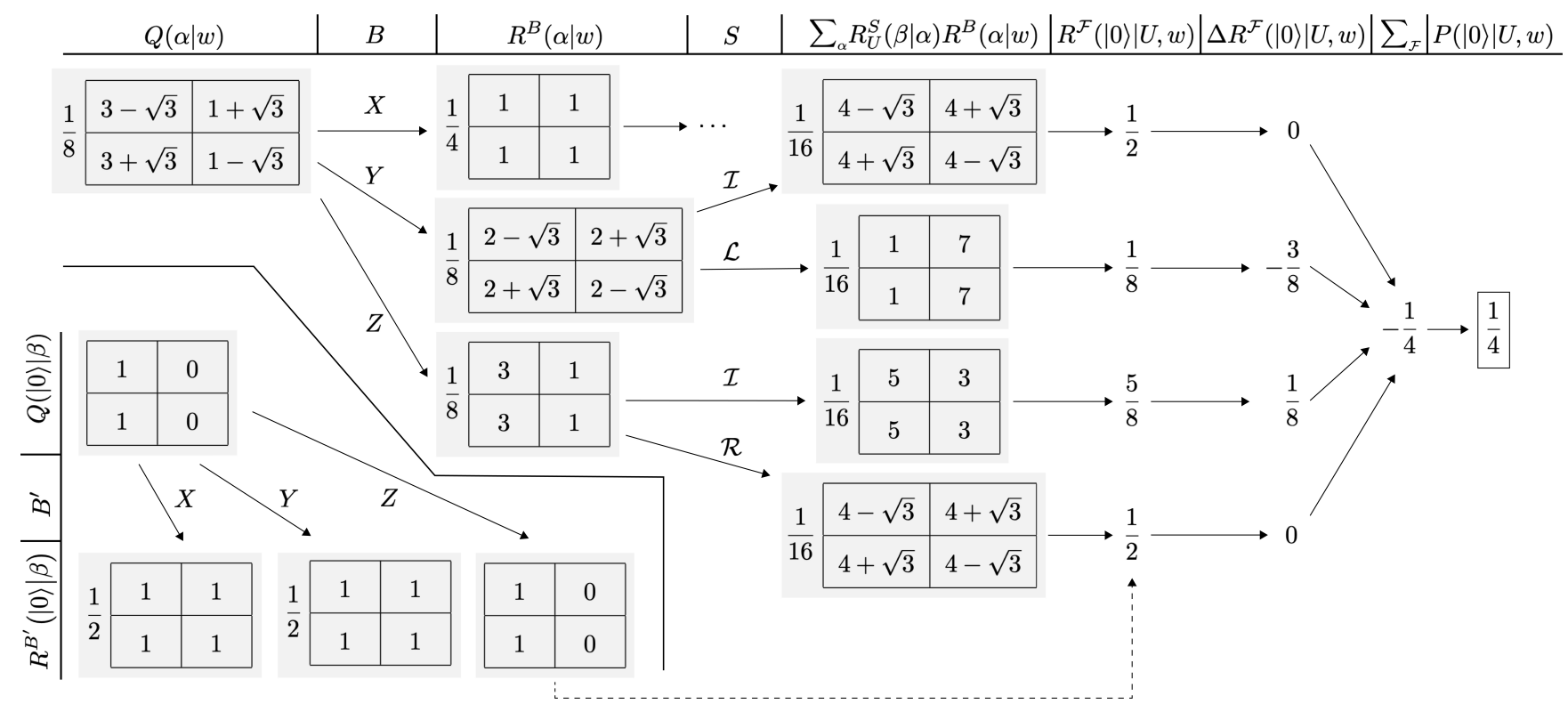

Figure 3: The steps leading from quantum distributions $(Q$ 's) to epistemically restricted classical probability distributions $(R$ 's), and then to a final probabilistic prediction $(P)$. The top line contains labels for the objects contained in imagined columns below. Under these labels, and starting in the top left with the Wigner function for a specific state, we follow the flow of arrows corresponding to specific choices of classical frameworks until we reach the only nonuniform premeasurement classical states. These lie in the column labeled $\sum_{\alpha} R_{U}^{S}(\beta \mid \alpha) R^{B}(\alpha \mid w)$. Separately, the bottom left section shows how quantum measurement distributions are related to epistemically resricted measurement distributions by a choice of measurement framework. The measurement distribution consistent with a choice of classical measurement framework $Z$ leads to a sum over the left column of the premeasurement state. This choice is indicated with the dashed arrow at the bottom. A sum of the nonrandom parts of the epistemically restricted classical probabilities $\left(\Delta R^{\mathcal{F}}(|0\rangle \mid U, w)\right.$ 's) added to the "random part" $(1 / 2)$ gives a probabilistic prediction in agreement with the Born rule. Note that there are many frameworks leading to the uniform distribution, as indicated by the "..." in the fourth column, that have no effect on the final prediction.

with $\beta$ and columns with $\alpha$. For the three legal symplectic transformations $\mathcal{I}, \mathcal{R}$, and $\mathcal{L}$, we find

$$
\begin{aligned}
& R_{U}^{\mathcal{I}}=\frac{1}{4}\left(\begin{array}{llll}
1 & 0 & 3 & 0 \\
0 & 1 & 0 & 3 \\
3 & 0 & 1 & 0 \\
0 & 3 & 0 & 1
\end{array}\right), \\
& R_{U}^{\mathcal{R}}=\frac{1}{8}\left(\begin{array}{llll}
2+\sqrt{3} & 2+\sqrt{3} & 2-\sqrt{3} & 2-\sqrt{3} \\
2-\sqrt{3} & 2-\sqrt{3} & 2+\sqrt{3} & 2+\sqrt{3} \\
2-\sqrt{3} & 2-\sqrt{3} & 2+\sqrt{3} & 2+\sqrt{3} \\
2+\sqrt{3} & 2+\sqrt{3} & 2-\sqrt{3} & 2-\sqrt{3}
\end{array}\right), \\
& R_{U}^{\mathcal{L}}=\frac{1}{8}\left(\begin{array}{llll}
2-\sqrt{3} & 2+\sqrt{3} & 2+\sqrt{3} & 2-\sqrt{3} \\
2-\sqrt{3} & 2+\sqrt{3} & 2+\sqrt{3} & 2-\sqrt{3} \\
2+\sqrt{3} & 2-\sqrt{3} & 2-\sqrt{3} & 2+\sqrt{3} \\
2+\sqrt{3} & 2-\sqrt{3} & 2-\sqrt{3} & 2+\sqrt{3}
\end{array}\right) .
\end{aligned}
$$

In a single classical experiment, a transformed epistemic state is given by

$$
\sum_{\alpha} R_{U}^{S}(\beta \mid \alpha) R^{B}(\alpha \mid w)
$$

This calculation is conveniently accomplished by using the matrix form of $R_{U}^{S}(\beta \mid \alpha)$ and a vectorized form of
$R^{B}(\alpha \mid w)$. Here are the only nonuniform distributions for transformed states a classical experimenter may find depending on a choice of framework:

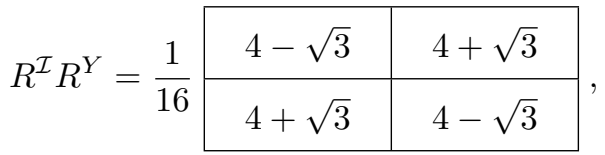

$$
\begin{aligned}
& R^{\mathcal{L}} R^{Y}=\frac{1}{16} \begin{array}{|c|c|}
\hline 1 & 7 \\
\hline 1 & 7 \\
\hline
\end{array} \\
& R^{\mathcal{I}} R^{Z}=\frac{1}{16} \begin{array}{|l|l|}
\hline 5 & 3 \\
\hline 5 & 3 \\
\hline
\end{array}
\end{aligned}
$$

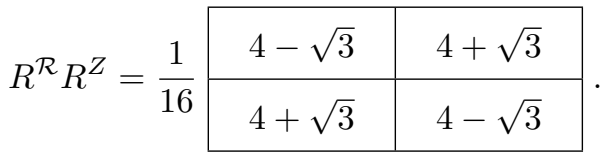

We now have the full collection of final states an experimenter may find. Remember that there are many unlisted ones that result in the uniform distribution.

We had set out to calculate the probability of a pho- 
ton being found in path $|0\rangle$ of the interferometer after the beamsplitter. In our formalism, this outcome is equivalent to the experimenter choosing the measurement framework $B^{\prime}=Z$ corresponding to the vertical striation and finding the system in the left line of that striation. This is because the effect $E=|0\rangle\langle 0|$ is represented by the measurement function given by Eq. (55):

$$
Q(E \mid \beta)=\begin{array}{|l|l|}
\hline 1 & 0 \\
\hline 1 & 0 \\
\hline
\end{array}
$$

We calculate $R^{B}(E \mid \beta)$ for the different frameworks using Eq. (59) leading to

$$
\begin{aligned}
& R^{X}(E \mid \beta)=\frac{1}{2} \begin{array}{|l|l|}
\hline 1 & 1 \\
\hline 1 & 1 \\
\hline
\end{array} \\
& R^{Y}(E \mid \beta)=\frac{1}{2} \begin{array}{|l|l|}
\hline 1 & 1 \\
\hline 1 & 1 \\
\hline
\end{array} \\
& R^{Z}(E \mid \beta)=\begin{array}{|l|l|}
\hline 1 & 0 \\
\hline 1 & 0 \\
\hline
\end{array}
\end{aligned}
$$

When one calculates the classical predictions given by an equation such as

$$
R^{B^{\prime}, S, B}(E \mid U, w)=\sum_{\beta, \alpha} R^{B^{\prime}}(E \mid \beta) R_{\mathcal{E}}^{S}(\beta \mid \alpha) R^{B}(\alpha \mid w),
$$

the choice of $B^{\prime}=Z$ clearly results in the sum over the points in left vertical line of the premeasurement epistemic state (such as those given in Eq. (100)). Meanwhile, a choice of $B^{\prime}=X$ or $Y$ will always result in a probability of $1 / 2$ for $E=|0\rangle\langle 0|$.

The marginal probabilities for the left vertical line of each phase space distribution in Eqs. (100) are 1/2, $1 / 8,5 / 8$, and $1 / 2$, respectively. We sum the nonrandom parts of these to get $0-3 / 8+1 / 8+0=-1 / 4$. Adding the random part back in, we have $-1 / 4+1 / 2=1 / 4$; this is the probability of the photon being measured in path $|0\rangle$ as predicted by the collection of classical experiments. By construction, it is consistent with the Born rule.

\section{Prime-power dimensions}

Consider a set of $n$ basic systems - we will call them particles - each having odd prime Hilbert-space dimension $r$. So $d=r^{n}$. For the phase space of this system, we could consider using the $2 n$-dimensional vector space over $\mathbb{Z}_{r}$. However, we find it more convenient to use the two-dimensional vector space over the finite field with $d$ elements, $\mathbb{F}_{d}$. (Note that for $n>1$, the elements of this field cannot be identified with ordinary integers. They are more abstract objects, with addition and multiplication rules satisfying the requirements of a field.) A point $\alpha$ in this phase space still has two components $\alpha_{q}$ and $\alpha_{p}$, but each of these components is now an element of $\mathbb{F}_{d}$. The concepts of "line" and "parallel lines" are defined exactly as in Section 2, and because of the field properties, it is still the case that any two nonparallel lines intersect in exactly one point. As before, there are $d+1$ striations, each consisting of $d$ parallel lines.

It is worth reviewing a few features of finite fields [56]. First, a basis for the field $\mathbb{F}_{r^{n}}$ is an ordered set of $n$ field elements $\left\{e_{j}\right\}$ such that any field element $x$ can be written as a linear combination of the $e_{j}$ 's with coefficients in $\mathbb{F}_{r}$; that is, $x=\sum_{j} x_{j} e_{j}$ with $x_{j} \in \mathbb{F}_{r}$. (When $r$ is prime, the field $\mathbb{F}_{r}$ is the same as $\mathbb{Z}_{r}$.) If we represent a field element $x$ as a column vector with entries $x_{j}$, then multiplication by any field element can be represented as ordinary matrix multiplication. In this way, we can also represent any field element $x$ as a matrix $M_{x}$ with entries in $\mathbb{F}_{r}$. The trace of this matrix is independent of the chosen basis and is called the trace of $x$ :

$$
\operatorname{Tr}_{\mathbb{F}_{d} / \mathbb{F}_{r}}(x)=\operatorname{tr} M_{x}
$$

where we use the subscript $\mathbb{F}_{d} / \mathbb{F}_{r}$ to indicate that the field trace is a function from $\mathbb{F}_{d}$ to $\mathbb{F}_{r}$. In what follows we will use "Tr" in place of " $\operatorname{Tr}_{\mathbb{F}_{d} / \mathbb{F}_{r}}$ " until the final paragraph of this section, where we need to distinguish two field traces. The trace is a linear function: (i) For any $x \in \mathbb{F}_{d}$ and $a \in \mathbb{F}_{r}, \operatorname{Tr}(a x)=a \operatorname{Tr}(x)$. (ii) For any $x, y \in \mathbb{F}_{d}, \operatorname{Tr}(x+y)=\operatorname{Tr}(x)+\operatorname{Tr}(y)$. The trace can also be expressed as

$$
\operatorname{Tr}(x)=x+x^{r}+x^{r^{2}}+\cdots+x^{r^{n-1}},
$$

which avoids any mention of a basis. Finally, for every basis $\left\{e_{j}\right\}$, there is a unique dual basis $\left\{\tilde{e}_{j}\right\}$, defined by the property that

$$
\operatorname{Tr}\left(\tilde{e}_{i} e_{j}\right)=\delta_{i j} .
$$

We use the expansion in a basis to write a phase-space coordinate such as $\alpha_{q}$ in a way that gives each of the $n$ particles its own phase-space variable. Let us choose a basis $\left\{e_{j}\right\}$ to associate with the horizontal component $\alpha_{q}$. In the expansion

$$
\alpha_{q}=\alpha_{q 1} e_{1}+\cdots+\alpha_{q n} e_{n},
$$

we think of $\alpha_{q j}$ (which is in $\mathbb{F}_{r}$ ) as the horizontal phasespace coordinate of the $j$ th particle. For the vertical component $\alpha_{p}$, we use the dual basis:

$$
\alpha_{p}=\alpha_{p 1} \tilde{e}_{1}+\cdots+\alpha_{p n} \tilde{e}_{n} .
$$


The reason for this choice will become evident shortly.

As the Wigner function for this system, we use the definition proposed by Vourdas [35] and by Klimov and Muñoz [34]. The phase-point operators look very much like the ones defined in Eq. (3), but with a field trace in the exponent of $\omega$, where $\omega$ is now equal to $e^{2 \pi i / r}$ :

$$
\left(A_{\alpha}\right)_{k l}=\delta_{2 \alpha_{q}, k+l} \omega^{\operatorname{Tr}\left[\alpha_{p}(k-l)\right]} .
$$

Here $k$ and $l$, like $\alpha_{q}$ and $\alpha_{p}$, are elements of $\mathbb{F}_{d}$. The Wigner function is defined in the same way as before in terms of the phase-point operators:

$$
Q(\alpha \mid w)=\frac{1}{d} \operatorname{tr}\left(w A_{\alpha}\right)
$$

We can understand the phase-point operators better by expanding the phase-space coordinates in their respective bases. Since $k$ and $l$ label the vectors of the standard basis, which is associated with the horizontal coordinate, we expand $k$ and $l$ in the basis $\left\{e_{j}\right\}$ associated with that coordinate. In Eq. (109), because $(k-l)$ is expanded in the basis $\left\{e_{j}\right\}$ while $\alpha_{p}$ is expanded in the dual basis $\left\{\tilde{e}_{j}\right\}$, the exponent of $\omega$ becomes simply

$$
\alpha_{p 1}\left(k_{1}-l_{1}\right)+\cdots+\alpha_{p n}\left(k_{n}-l_{n}\right) .
$$

Indeed, one sees that the entire expression factors, so that

$$
A_{\alpha}=A_{\alpha_{1}} \otimes \cdots \otimes A_{\alpha_{n}},
$$

where $\alpha_{j}=\left(\alpha_{q j}, \alpha_{p j}\right)$ and $A_{\alpha_{j}}$ is the usual phase-point operator of Eq. (3) for an $r \times r$ phase space. (See the related analyses in Refs. [34-36].) In this form, the phase-point operators look like those of Ref. [24], where the phase space is a $2 n$-dimensional vector space over $\mathbb{Z}_{r}$. However, there is a conceptual difference between the two formalisms, concerning the way the phase space is partitioned to define marginal probabilities. In the higher-dimensional phase space, it is natural to consider an $n$-dimensional "slice," which is an ordered $n$-tuple of lines from the $r \times r$ phase spaces of the individual particles. The sum of the phase-point operators over such a slice is the projection operator onto a pure product state. In contrast, the sum of the $A_{\alpha}$ 's over a line in the two-dimensional phase space over $\mathbb{F}_{d}$ is the projection operator onto a pure state that may well be entangled. As in the prime-dimensional case, each of the $d+1$ striations defines an orthogonal basis for the Hilbert space, and these $d+1$ bases are mutually unbiased.

Once we adopt this two-dimensional phase space, with the phase-point operators given by Eq. (109), one can check that every step of our arguments in Sections 3 and 4 goes through exactly as before. In particular, we use the fact that, as in the odd prime case, every symplectic matrix $S$ is legal in the sense that there exists a unitary transformation $U_{S}$ such that [47]

$$
U_{S} A_{\alpha} U_{S}^{\dagger}=A_{S \alpha}
$$

The displacement operators, defined by

$$
D_{\alpha}=D_{\alpha_{1}} \otimes \cdots \otimes D_{\alpha_{n}}
$$

still satisfy Eq. (8). It is simply that the symbols representing phase-space points are now elements of $\mathbb{F}_{d}^{2}$.

Thus, for an experiment consisting of a preparation, channel, and measurement, we can still take as our framework the triple $\mathcal{F}=\left(B^{\prime}, S, B\right)$, where $B$ and $B^{\prime}$ are striations and $S$ is a symplectic matrix. The classical experiment is still described by $R^{B}(\alpha \mid w), R_{\mathcal{E}}^{S}(\beta \mid \alpha)$, and $R^{B^{\prime}}(E \mid \beta)$, all given by the same formulas as before. And the actual quantum prediction for the probability of the outcome $E$ is still given by

$$
\Delta P(E \mid \mathcal{E}, w)=\frac{1}{\mathcal{Z}} \sum_{\mathcal{F}} \Delta R^{\mathcal{F}}(E \mid \mathcal{E}, w)
$$

with $\mathcal{F}=\left(B^{\prime}, S, B\right)$ and the classical prediction given by

$$
R^{\mathcal{F}}(E \mid \mathcal{E}, w)=\sum_{\beta, \alpha} R^{B^{\prime}}(E \mid \beta) R_{\mathcal{E}}^{S}(\beta \mid \alpha) R^{B}(\alpha \mid w) .
$$

The redundancy factor $\mathcal{Z}$ is again equal to $d$ if we use the full symplectic group. If there exists a smaller "minimal reconstructing set," then we could use that set and have $\mathcal{Z}=1$. We are not aware of work either proving or disproving the existence of such a special set of symplectic matrices for any value of $d$ equal to $r^{n}$ with $r$ an odd prime and $n>1$.

Suppose we have the complete description of a preparation for a system of $n$ particles, each with odd-prime Hilbert-space dimension $r$. That is, we have all the probability distributions $R^{B}(\alpha \mid w)$. Is there a simple way to obtain from this description a similar description of the effective preparation of just one of the particles, say, the first one? In Hilbert space language, we would take a partial trace of the whole system's density matrix. What does this partial trace look like in our formalism?

We begin by addressing this question at the level of the Wigner function. In terms of the Wigner function, the density matrix of the whole system is given by

$$
\begin{aligned}
w & =\sum_{\alpha} Q(\alpha \mid w) A_{\alpha} \\
& =\sum_{\alpha} Q(\alpha \mid w) A_{\alpha_{1}} \otimes \cdots \otimes A_{\alpha_{n}} .
\end{aligned}
$$

Taking the partial trace of $w$ to get the state $w_{1}$ of the first particle, we have

$$
w_{1}=\operatorname{tr}_{2, \ldots, n} w=\sum_{\alpha_{1}}\left[\sum_{\alpha_{2}, \ldots, \alpha_{n}} Q\left(\alpha_{1}, \ldots, \alpha_{n} \mid w\right)\right] A_{\alpha_{1}}
$$


Evidently the quantity in square brackets is playing the role of the Wigner function for the single-particle state $w_{1}$. That is, we can write

$$
Q\left(\alpha_{1} \mid w_{1}\right)=\sum_{\alpha_{2}, \ldots, \alpha_{n}} Q\left(\alpha_{1}, \ldots, \alpha_{n} \mid w\right) .
$$

Thus as far as the Wigner function is concerned, we take the partial trace by summing over the phase-space coordinates of the traced-out particles. Since $\alpha_{1}$ is a vector in $\mathbb{F}_{r}^{2}$, it is perfectly appropriate for labeling the phase-space points of particle 1. (Note: We are not making a similar claim for all examples of partial tracing. If we trace over all but the first two particles, for example, we are left with $\alpha_{1}$ and $\alpha_{2}$, but the expression $\alpha_{q 1} e_{1}+\alpha_{q 2} e_{2}$ does not make sense in the field $\mathbb{F}_{r^{2}}$, because $e_{1}$ and $e_{2}$ are not elements of that field.)

We now translate the Wigner-function picture into the language of the $R^{B}$ 's. We begin with a combination of Eqs. (32) and (34):

$$
R^{B}(\alpha \mid w)=\frac{1}{d} \sum_{\beta \in \ell} Q(\beta \mid w),
$$

where $\ell$ is the line in $B$ that contains the point $\alpha$. Let us define $R^{B}\left(\alpha_{1} \mid w_{1}\right)$ by summing over $\alpha_{2}, \ldots, \alpha_{n}$.

$$
R^{B}\left(\alpha_{1} \mid w_{1}\right)=\sum_{\alpha_{2}, \ldots, \alpha_{n}} R^{B}(\alpha \mid w) .
$$

The notation indicating that this expression depends only on $w_{1}$ (and not on any other aspects of the full density matrix $w$ ) is justified by the relation (120) and the fact that $Q\left(\alpha_{1} \mid w_{1}\right)$ depends only on $w_{1}$. Now we use Eq. (37) (which is still valid in this new context) to write

$$
\begin{aligned}
Q\left(\alpha_{1} \mid w_{1}\right) & =\sum_{\alpha_{2}, \ldots, \alpha_{n}} Q\left(\alpha_{1}, \ldots, \alpha_{n} \mid w\right) \\
& =\sum_{\alpha_{2}, \ldots, \alpha_{n}}\left[\sum_{B} R^{B}\left(\alpha_{1}, \ldots, \alpha_{n} \mid w\right)-\frac{1}{d}\right] \\
& =\sum_{B} R^{B}\left(\alpha_{1} \mid w_{1}\right)-\frac{r^{2(n-1)}}{r^{n}} .
\end{aligned}
$$

With the substitutions

$$
\begin{gathered}
Q\left(\alpha_{1} \mid w_{1}\right)=\Delta Q\left(\alpha_{1} \mid w_{1}\right)+\frac{1}{r^{2}}, \\
R^{B}\left(\alpha_{1} \mid w_{1}\right)=\Delta R^{B}\left(\alpha_{1} \mid w_{1}\right)+\frac{1}{r^{2}},
\end{gathered}
$$

we find that all the additive constants drop out, and we are left with

$$
\Delta Q\left(\alpha_{1} \mid w_{1}\right)=\sum_{B} \Delta R^{B}\left(\alpha_{1} \mid w_{1}\right) .
$$

Now, Eq. (124) is not in our standard form for an $r$-dimensional particle, because the superscript $B$ still ranges over all the striations of the large phase space $\mathbb{F}_{d}^{2}$. However, we can convert this expression to our standard form via the following facts, which we prove in Appendix C. (i) If the slope $m$ of the striation $B$ is of the form $m=m_{1} e_{1}^{-1} \tilde{e}_{1}$ with $m_{1} \in \mathbb{Z}_{r}$, or if the slope is infinite, then $R^{B}\left(\alpha_{1} \mid w_{1}\right)=R^{B_{1}}\left(\alpha_{1} \mid w_{1}\right)$, where $B_{1}$ is the striation in $\mathbb{Z}_{r}^{2}$ whose slope is $m_{1}$, or infinity if $m=\infty$. (ii) If the slope $m$ is of the form $m=$ $\sum_{j} m_{j} e_{1}^{-1} \tilde{e}_{j}$ with $m_{j} \in \mathbb{Z}_{r}$ and $m_{j} \neq 0$ for some $j \neq$ 1 , then $\Delta R^{B}\left(\alpha_{1} \mid w_{1}\right)=0$, so those striations do not contribute to the sum in Eq. (124). This leaves us with

$$
\Delta Q\left(\alpha_{1} \mid w_{1}\right)=\sum_{B_{1}} \Delta R^{B_{1}}\left(\alpha_{1} \mid w_{1}\right)
$$

which is in our standard form. In this way, we can go from a description of the $n$-particle system to a description of the state of the first particle. That is, we have a way of "tracing out" the other $n-1$ particles, and the resulting picture accords with our earlier treatment of a particle with a prime-dimensional Hilbert space.

We illustrate these ideas by working out a specific example in detail. Consider a pair of qutrits in the pure, entangled state

$$
|\psi\rangle=\frac{1}{2 \sqrt{2}}(|00\rangle+|01\rangle+|10\rangle+|11\rangle)+\frac{1}{\sqrt{2}}|22\rangle .
$$

Its Wigner function will be a real function on the $9 \times 9$ phase space $\mathbb{F}_{9}^{2}$. We construct the field $\mathbb{F}_{9}$ by identifying a quadratic polynomial, with coefficients in $\mathbb{F}_{3}$, that has no roots in $\mathbb{F}_{3}$. Such a polynomial is $x^{2}+1$. Let us then define $\xi$ to be such that $\xi^{2}+1=0$; that is, $\xi^{2}=2$. (This is analogous to defining the imaginary unit $i$ to satisfy $i^{2}+1=0$, which no real number satisfies.) A general element of $\mathbb{F}_{9}$ is then of the form $a+b \xi$ with $a, b \in \mathbb{F}_{3}$.

In order to treat our $d=9$ system as two distinct qutrits, we choose a field basis in which to write the horizontal phase-space coordinate. Let us choose the basis $\left(e_{1}, e_{2}\right)=(1, \xi)$. One finds that the dual basis is $\left(\tilde{e}_{1}, \tilde{e}_{2}\right)=(2, \xi)$, and we use this dual basis to expand the vertical phase-space coordinate. With these conventions, we show here the Wigner function of the state $|\psi\rangle$ given in Eq. (126). 
result is

\begin{tabular}{|c|c|c|c|c|c|c|c|c|c|c|}
\hline $1+2 \xi$ & 22 & -1 & 5 & -1 & 5 & -1 & -1 & -1 & -1 & 5 \\
\hline $1+\xi$ & 21 & 5 & -1 & -1 & -1 & 5 & -1 & -1 & -1 & 5 \\
\hline 1 & 20 & -1 & -1 & 2 & -1 & -1 & 2 & -1 & \begin{tabular}{|l|}
-1 \\
\end{tabular} & 2 \\
\hline $2+2 \xi$ & 12 & 5 & -1 & -1 & -1 & 5 & -1 & -1 & -1 & 5 \\
\hline $2+\xi$ & 11 & -1 & 5 & -1 & 5 & -1 & -1 & -1 & -1 & 5 \\
\hline 2 & 10 & -1 & -1 & 2 & -1 & -1 & 2 & -1 & -1 & 2 \\
\hline $2 \xi$ & 02 & -1 & -1 & -1 & -1 & -1 & -1 & 2 & 2 & 2 \\
\hline$\xi$ & 01 & -1 & -1 & -1 & -1 & -1 & -1 & 2 & 2 & 2 \\
\hline 0 & 00 & 5 & 5 & 2 & 5 & 5 & 2 & 2 & 2 & 8 \\
\hline & & 00 & 01 & 02 & 10 & 11 & 12 & 20 & 21 & 22 \\
\hline & & 0 & $\xi$ & $2 \xi$ & 1 & $1+\xi$ & $+2 \xi$ & 2 & $2+\xi$ & $2+2 \xi$ \\
\hline
\end{tabular}

The first line of labels along the horizontal axis shows the pairs $\left(\alpha_{q 1}, \alpha_{q 2}\right)$. The line below it shows the field values $\alpha_{q}=\alpha_{q 1} e_{1}+\alpha_{q 2} e_{2}$. Similarly, along the vertical axis we have $\left(\alpha_{p 1}, \alpha_{p 2}\right)$ and $\alpha_{p}=\alpha_{p 1} \tilde{e}_{1}+\alpha_{p 2} \tilde{e}_{2}$.

From the Wigner function, we can construct the probability distribution $R^{B}(\alpha \mid w)$ for any striation $B$. Here we choose the striation with slope $m=2$. So we sum the Wigner function over each line with slope 2 , and we spread this sum uniformly over the line to get $R^{B}(\alpha \mid w)$. Here is the result:

\begin{tabular}{|c|c|c|c|c|c|c|c|c|c|c|}
\hline $1+2 \xi$ & 22 & 0 & 1 & 1 & 4 & 0 & 0 & 0 & 1 & 1 \\
\hline $1+\xi$ & 21 & 1 & 0 & 1 & 0 & 4 & 0 & 1 & 0 & 1 \\
\hline 1 & 20 & 1 & 1 & 0 & 0 & 0 & 4 & 1 & 1 & 0 \\
\hline $2+2 \xi$ & 12 & 4 & 0 & 0 & 0 & 1 & 1 & 0 & 1 & 1 \\
\hline $2+\xi$ & 11 & 0 & 4 & 0 & 1 & 0 & 1 & 1 & 0 & 1 \\
\hline 2 & 10 & 0 & 0 & 4 & 1 & 1 & 0 & 1 & 1 & 0 \\
\hline $2 \xi$ & 02 & 0 & 1 & 1 & 0 & 1 & 1 & 4 & 0 & 0 \\
\hline$\xi$ & 01 & 1 & 0 & 1 & 1 & 0 & 1 & 0 & 4 & 0 \\
\hline 0 & 00 & 1 & 1 & 0 & 1 & 1 & 0 & 0 & 0 & 4 \\
\hline & & 00 & 01 & 02 & 10 & 11 & 12 & 20 & 21 & 22 \\
\hline & & 0 & $\xi$ & $2 \xi$ & 1 & $1+\xi$ & $1+2 \xi$ & 2 & $2+\xi$ & $+2 \xi$ \\
\hline
\end{tabular}

Now, is this striation, with slope $m=2$, a striation that contributes to Eq. (125)? The test is to expand $m$ in the basis $\left(e_{1}^{-1} \tilde{e}_{1}, e_{1}^{-1} \tilde{e}_{2}\right)$ - that is, to find $m_{1}$ and $m_{2}$ in $m=m_{1} e_{1}^{-1} \tilde{e}_{1}+m_{2} e_{1}^{-1} \tilde{e}_{2}$-and to see whether $m_{2}$ is zero. If it is, then this striation does contribute to Eq. (125), and the corresponding striation in the small phase space $\mathbb{F}_{3}^{2}$ is the one with slope $m_{1}$. Upon doing the expanding, we get $m=2=1(2)+0(\xi)$. So indeed, this striation does contribute, and the corresponding striation has slope $m_{1}=1$.

We can see how this works out by summing $R^{B}(\alpha \mid w)$ over each of the $3 \times 3$ grids bounded by thick lines. The

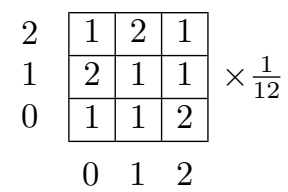

One can check that this is indeed the correct distribution $R^{B_{1}}\left(\alpha_{1} \mid w_{1}\right)$ for the reduced density matrix $w_{1}=\operatorname{tr}_{2} w$ and for the striation $B_{1}$ with slope $m_{1}=1$.

For the above construction, it is not necessary that the dimension of each individual particle be prime. Suppose we have a system of $n$ particles, each of which has Hilbert-space dimension $r$, where $r$ is a nontrivial power of an odd prime $p$. So $d=r^{n}$ with $r=p^{s}$. The phase space for a single particle will be $\mathbb{F}_{r}^{2}$. To get the phase space for the whole system, we form the extension field $\mathbb{F}_{r^{n}}$. This field is none other than $\mathbb{F}_{d}$-there is only one field with $d$ elements - but we wish to think of the field as an extension of $\mathbb{F}_{r}$. To obtain phasespace coordinates for the individual particles, we expand the horizontal coordinate $\alpha_{q}$ in a basis $\left(e_{1}, \ldots, e_{n}\right)$. That is, $\alpha_{q}=\sum_{j} \alpha_{q j} e_{j}$, where $\alpha_{q j}$ is an element of $\mathbb{F}_{r}$. We expand the vertical coordinate $\alpha_{p}$ in the dual basis $\left(\tilde{e}_{1}, \ldots, \tilde{e}_{n}\right)$. Here the field trace used to define the dual is $\operatorname{Tr}_{\mathbb{F}_{d} / \mathbb{F}_{r}}$, a function from $\mathbb{F}_{d}$ to $\mathbb{F}_{r}$ (and not a function from $\mathbb{F}_{d}$ to $\mathbb{F}_{p}$ ). It can be defined either by Eq. (104) or by Eq. (105). We ultimately take the absolute trace, taking values in $\mathbb{F}_{p}$, in the definition of the phase-point operators, which we now write as

$$
\left(A_{\alpha}\right)_{k l}=\delta_{2 \alpha_{q}, k+l} \omega^{\operatorname{Tr}_{\mathbb{F}_{d} / \mathbb{F}_{p}}\left[\alpha_{p}(k-l)\right]}
$$

with $\omega$ equal to $e^{2 \pi i / p}$. With these definitions, one finds that everything we have done for odd prime values of $r$ continues to work. In particular, starting with a state of $n$ particles as represented by a collection of $R^{B}$ distributions, we can take appropriate sums over the coordinates of the last $n-1$ particles to get the state of the first particle. That is, our method of taking the partial trace remains valid in this more general setting.

\section{Challenges}

The formalism we have developed cannot be immediately extended to all values of the Hilbert-space dimension $d$. In this paper we have relied heavily, though often implicitly, on the properties of finite fields, and when $d$ is not a prime power, there exists no finite field having exactly $d$ elements. In the present section, we describe some of the difficulties one encounters for values of $d$ other than those we have considered.

We begin with a bit of parameter counting. A general unital channel requires $\left(d^{2}-1\right)^{2}$ parameters for its 
specification. For a fixed symplectic matrix $S$, the probability distribution $P_{\mathcal{E}}^{S}(\delta)$ provides $d^{2}-1$ parameters, since there are $d^{2}$ values of $\delta$ but the probabilities must sum to unity. So we need at least $d^{2}-1$ legal symplectic matrices in order for the whole set of $P^{S}$ 's to have a chance of representing a general unital channel. A minimal reconstructing set comprises precisely this many matrices.

Now consider a composite dimension that is not a prime power, such as $d=15=3 \cdot 5$. By using as our phase space the Cartesian product of $\mathbb{Z}_{3}^{2}$ and $\mathbb{Z}_{5}^{2}$, we can in fact generalize our treatment of preparations and measurements to this case, for example representing a preparation $w$ by probability distributions $R^{B_{1}, B_{2}}\left(\alpha_{1}, \alpha_{2} \mid w\right)$, where the $B$ 's and $\alpha$ 's refer to striations and points in $\mathbb{Z}_{3}^{2}$ and $\mathbb{Z}_{5}^{2}$. (We plan to present the details of this generalization in a future paper.) Our main difficulty arises in the description of a channel. It is perfectly sensible to speak of the group of symplectic matrices with entries in $\mathbb{Z}_{15}$ [38]. This group factorizes into the symplectic groups represented by matrices with entries in $\mathbb{Z}_{3}$ and $\mathbb{Z}_{5}$. If we try to form a minimal reconstructing set, starting with the minimal reconstructing sets for dimensions 3 and 5 , we end up with only $\left(3^{2}-1\right)\left(5^{2}-1\right)$ matrices, fewer than the $\left(15^{2}-1\right)$ that we need. We could try using the full symplectic group, which has more than enough matrices, but even then, one can show that it is impossible to reconstruct $Q_{\mathcal{E}}(\beta \mid \alpha)$ from the whole set of distributions $P_{\mathcal{E}}^{S_{1}, S_{2}}\left(\delta_{1}, \delta_{2}\right)$. This fact does not imply that there is no extension of our work to the case $d=15$, only that there is no obvious, immediate extension.

Dimensions that are powers of 2 , as when the system is a collection of qubits, also present a challenge, even though there does exist a field of order $2^{n}$ for every positive integer $n$. In this case, the challenge comes from the scarcity of legal symplectic matrices. We have already seen that for $d=2$, only three of the six symplectic matrices are legal in the sense that they correspond to unitary transformations of the phase-point operators (Eq. (20)). This is not a problem for $d=2$, because three is precisely the number of symplectic matrices we need. However, it is already a problem for $d=4$, e.g., for a two-qubit system.

In dimension 4 , it is certainly possible to use as a phase space the two-dimensional vector space over the field $\mathbb{F}_{4}$, in analogy with what we have done in Section 7. However, in contrast to the case of odd-prime-power dimension, there is no single, natural choice of phasepoint operators for dimension 4 . Within the framework of Ref. [32], there are, for $d=4$, four truly distinct ways of defining the phase-point operators (four similarity classes, in the terminology of Ref. [32]). There are exactly 60 symplectic $2 \times 2$ matrices with entries in $\mathbb{F}_{4}$, and for any given definition of the phase-point operators, one can check to see how many of these symplectic matrices are legal. One finds that for two of the four possible definitions, there are only three legal matrices, and for the other two, there are only five legal matrices. But we need $4^{2}-1=15$ legal matrices $S$ to characterize a general unital channel.

There are various avenues one could follow toward generalizing our formalism. One possibility, which might help in trying to build a formalism for $d=15$ out of the cases $d=3$ and $d=5$, is to extend the list of classical frameworks in our description of a channel. In Fig. 2, one can discern in the $4 \times 4$ grids a set of three striation-like partitions, for which each "line" of one partition intersects each line of any other partition in exactly one point. It is possible to add two other partitions of the grid and still retain this relation between any pair of partitions. The new ones would consist of the horizontal and vertical lines. Should we look for a way of including these other two partitions in our picture? (For this single-qubit case, the partitions can in fact be identified with striations of $\mathbb{F}_{4}^{2}$.) Alternatively, and especially for dealing with even values of $d$, one could try switching from a $d \times d$ phase space to a $2 d \times 2 d$ phase space $[26,27]$. This phase space and the associated Wigner function were designed specifically to accommodate even values of the Hilbert-space dimension.

A less elegant solution is simply to note that every system with a finite-dimensional Hilbert space can be accommodated within any Hilbert space of higher dimension. So, if we want to treat within our formalism a system of dimension 6 , we treat it as a system with dimension 7 . In this sense, what we have already done is sufficient for the quantum description of any discrete system.

The case of continuous phase space, applicable to a system with an infinite-dimensional Hilbert space, is clearly a case that needs to be considered. Much work has been done on the representation of quantum states in terms of the marginal distributions over such a phase space [7]. The generalization of our treatment of channels to continuous phase space will likely raise new questions. We plan to treat this case in future work.

\section{Conclusions}

We begin this section by writing down our partial formulation of quantum theory in a self-contained way, without reference to Hilbert space or quasiprobabilities.

First, rather than characterizing a discrete system by its Hilbert-space dimension, we adopt an operational definition of $d$ : it is the maximum number of outcomes of a repeatable measurement, that is, a measurement 
that yields the same outcome when repeated. In what follows, we consider only systems with odd-prime-power values of $d$. Also, we will now interpret the symbols $w, \mathcal{E}$ and $E$ purely operationally in the sense that $w$ refers solely to a preparation procedure, $\mathcal{E}$ to a physical channel, and $E$ to a measurement outcome. None of these symbols refers to an operator on Hilbert space or an operation on Hilbert-space operators. In these terms, we express the basics of operational quantum theory as follows.

A preparation $w$ is represented by a collection of probability distributions $R^{B}(\alpha \mid w)$, where $\alpha$ takes values in the $d \times d$ discrete phase space $\mathbb{F}_{d}^{2}$ and $B$ is a striation of that space. Each distribution $R^{B}$ must satisfy an epistemic constraint: it must be uniform over each line of the striation $B$.

A channel $\mathcal{E}$ is represented by a collection of conditional probability distributions $R_{\mathcal{E}}^{S}(\beta \mid \alpha)$, where $S$ is a symplectic matrix. Each such distribution must satisfy an epistemic constraint: it can depend only on the displacement $\delta=\beta-S \alpha$.

A measurement outcome $E$ is represented by a collection of conditional probability functions $R^{B^{\prime}}(E \mid \beta)$. Each such function must satisfy an epistemic constraint: it must be uniform over each line in the striation $B^{\prime}$.

In addition to the epistemic constraints, the $R$ functions describing each component of the experiment must also satisfy certain global constraints, as we have noted in the Introduction. For example, the distributions $R^{B}(\alpha \mid w)$ cannot be chosen independently of each other. These additional constraints are specified in Appendix D.

Once all the $R$ functions have been given, we compute, for each coherent framework, the classical probability of the outcome $E$. We do this using the ordinary rules of probability theory. If the experiment includes a single channel, this probability is

$$
R^{\mathcal{F}}(E \mid w)=\sum_{\beta, \alpha} R^{S B}(E \mid \beta) R^{S}(\beta \mid \alpha) R^{B}(\alpha \mid w) .
$$

We then compute the quantum probability of the outcome $E$ via the formula

$$
\Delta P(E \mid w)=\frac{1}{\mathcal{Z}} \sum_{\mathcal{F}} \Delta R^{\mathcal{F}}(E \mid w) .
$$

Though this description is self-contained, we again note that the constraints written down in Appendix D are not as simple as we would like. They are based on the analogous constraints on the Wigner function and the transition quasiprobabilities, and they appear simpler in that quasiprobabilistic setting than in our current setting. In a separate paper, we explore the idea that the global constraints may follow from a short list of principles [57]. Our central principle can be stated informally in this way: to find the probability functions representing the combination of two components of an experiment, sum the nonrandom parts of the classically expected results over every relevant framework. We show that when this idea is augmented with a few modest assumptions, we can at least reconstruct the quantum theory of a single qubit. We hope that these or similar principles prove to be sufficient to single out quantum theory when $d$ is a prime greater than 2 .

It is worth noting that with a complete collection of classical descriptions of an experiment, the description of the quantum experiment is always complete at every step. For example, at any point as we move horizontally through Fig. 3, we can translate the restricted classical descriptions into a quantum description, and it will be the correct quantum description of that phase of the experiment. Indeed, this fact is the basis of the central principle described in the preceding paragraph.

It is interesting also to think about how our formalism applies to quantum computation. We have seen how our construction can describe a quantum system with Hilbert space dimension equal to an odd prime power. A quantum computer consisting of $d$-dimensional units, with prime $d$, is such a system, so we can imagine a quantum computation implemented as a series of gates acting on a standard initial state of this system. Within a given framework, we could access the output statistics of one "branch" of the quantum computation by sampling from the initial state (a probability distribution $R^{B}(\alpha \mid w)$ over phase space) and carrying out the stochastic process defined by the transition probabilities $R_{\mathcal{E}_{j}}^{S_{j}}(\beta \mid \alpha)$ for the series of gates. However, to capture the full quantum computation, we would need to keep track of all the frameworks, and the number of frameworks grows exponentially both with the number of qudits and with the number of gates.

One can ask what error is incurred if we try to get away with considering only a subset of the frameworks and extrapolating from those results. It can certainly be the case that some frameworks are more important than others; for example, if one of our gates is the unitary transformation $U_{S}$ associated with a symplectic transformation $S$, then the framework indexed by that $S$ is the only one we need to consider for that gate. So there may be a clever way of limiting the number of frameworks. Our unusual rule for combining probabilities raises additional questions. For example, if we have the ability to sample from each of a set of probability distributions, can we efficiently simulate the effect of sampling from the distribution obtained by summing the nonrandom parts? And is there a sense in which this summation procedure substitutes for the negativity that has been identified as the key to the power of 
quantum computation [58-60]? We leave these questions for future investigations.

As we mentioned in the Introduction, we do not claim to know how to extract from our work an answer to the question: what is really going on? A simple classical theory with an epistemic constraint can be read as answering that question: what is really going on is that the system is always in one of the ontic states, but a measurement disturbs the system, and the epistemic constraint prevents the observer from ever knowing the system's true state. For the description we are adopting here, we have no such account of reality to offer. Each of our imagined classical observers might construct for themselves such an account - their computed probability $R^{\mathcal{F}}(E \mid w)$ can be perfectly well explained by a picture of the system jumping around in phase space - but we, who are assembling the predictions of all these classical observers, cannot explain the quantum probability $P(E \mid w)$ in this way. If we were forced to put our prediction algorithm into the standard probabilistic form (as in Eq. (131), for example), we would naturally be led to use quasiprobabilities; that is, we would return to the Wigner-function formulation of quantum theory. For us, the cost of a description based on ordinary probability distributions, as opposed to quasiprobabilities, is that we must use the rule (132), which, though mathematically simple and conceptually intriguing, is difficult to interpret as a way of combining probabilities. Indeed, if it were not for the constraints on the relationships among the $R$ distributions (Appendix $\mathrm{D}$ ), this rule for combining probabilities could easily yield a probability greater than 1 or less than 0 .

This is not to say that no ontological interpretation of our formalism can be found. Our picture lies outside the standard framework of ontological models [20], but generalizations of that framework have been proposed [61] and it is conceivable that one can construct a picture of reality that gives physical meaning to the mathematics we have presented here. As we have suggested above, we can reasonably hope to find a natural way of explaining the global constraints listed in Appendix D. What remains, then, is to find a physical interpretation of the enigmatic formula (132) by which the predictions from our many classical worlds are to be combined.

\section{A Proof of a Kronecker-delta equality}

Here we prove Eq. (52), which we copy here:

$$
\frac{1}{\mathcal{Z} d^{2}} \sum_{S, \delta} \delta_{\beta, S \alpha+\delta} \delta_{\beta^{\prime}, S \alpha^{\prime}+\delta}=\delta_{\beta \beta^{\prime}} \delta_{\alpha \alpha^{\prime}}+\frac{1}{d^{2}}\left(1-\delta_{\beta \beta^{\prime}}-\delta_{\alpha \alpha^{\prime}}\right)
$$

We start with the left-hand side (which we call "LHS" in what follows) and use one of the Kronecker deltas to do the sum over $\delta$. This gives us

$$
\mathrm{LHS}=\frac{1}{\mathcal{Z} d^{2}} \sum_{S} \delta_{S\left(\alpha^{\prime}-\alpha\right),\left(\beta^{\prime}-\beta\right)}
$$

We now use a nice property of the symplectic group $\operatorname{Sp}\left(2, \mathbb{F}_{d}\right)$ where $\mathbb{F}_{d}$ is any finite field, such as $\mathbb{Z}_{d}$ when $d$ is prime [48]: for any nonzero points $\mu$ and $\nu$ in phase space,

$$
\sum_{S} \delta_{S \mu, \nu}=d
$$

That is, as $S$ ranges over all symplectic matrices, $S \mu$ hits every nonzero point exactly $d$ times. The result is even simpler if, instead of using the full set of symplectic matrices, we use a "minimal reconstructing set," that is, a set of just $d^{2}-1$ symplectic matrices (out of $d\left(d^{2}-1\right)$ ) such that the difference between any two of them has nonzero determinant. One can see that this property guarantees (again with $\mu$ and $\nu$ nonzero) that

$$
\sum_{S} \delta_{S \mu, \nu}=1
$$

(If $\operatorname{det}\left(S_{1}-S_{2}\right) \neq 0$, it follows that for any nonzero $\alpha$, $\left(S_{1}-S_{2}\right) \alpha \neq 0$, so that $S_{1} \alpha \neq S_{2} \alpha$. Thus the $d^{2}-1$ distinct $S$ matrices must send $\alpha$ to the $d^{2}-1$ nonzero points.) We can summarize Eqs. (135) and (136) via our symbol $\mathcal{Z}$, which equals $d$ when we are using the whole symplectic group and equals 1 when we are using a minimal reconstructing set:

$$
\sum_{S} \delta_{S \mu, \nu}=\mathcal{Z}, \quad \mu, \nu \neq 0
$$

We now apply this fact to Eq. (134). If $\alpha$ equals $\alpha^{\prime}$, the sum over $S$ is zero unless $\beta$ equals $\beta^{\prime}$, in which case the sum is $\mathcal{Z}\left(d^{2}-1\right)$. If $\alpha$ is not equal to $\alpha^{\prime}$, the sum over $S$ is zero unless $\beta$ is not equal to $\beta^{\prime}$, in which case the sum is $\mathcal{Z}$, in accordance with Eq. (137). That is, we have

$$
\begin{aligned}
\text { LHS } & =\frac{1}{\mathcal{Z} d^{2}}\left[\delta_{\alpha \alpha^{\prime}} \delta_{\beta \beta^{\prime}} \mathcal{Z}\left(d^{2}-1\right)+\left(1-\delta_{\alpha \alpha^{\prime}}\right)\left(1-\delta_{\beta \beta^{\prime}}\right) \mathcal{Z}\right] \\
& =\delta_{\beta \beta^{\prime}} \delta_{\alpha \alpha^{\prime}}+\frac{1}{d^{2}}\left(1-\delta_{\beta \beta^{\prime}}-\delta_{\alpha \alpha^{\prime}}\right)
\end{aligned}
$$

which is what we wanted to prove. 


\section{B Distributing the $\Delta$ 's}

Here we prove a relationship that we call distributing the $\Delta$ 's. We will treat the case where a preparation, channel, and measurement are performed. We wish to show that

$$
\Delta\left[\sum_{\beta, \alpha} R^{B^{\prime}}(E \mid \beta) R_{\mathcal{E}}^{S}(\beta \mid \alpha) R^{B}(\alpha \mid w)\right]=\sum_{\beta, \alpha} \Delta R^{B^{\prime}}(E \mid \beta) \Delta R_{\mathcal{E}}^{S}(\beta \mid \alpha) \Delta R^{B}(\alpha \mid w) .
$$

We start by rewriting the left-hand side using two applications of our rule for $\Delta$. This gives

$$
\begin{aligned}
\Delta & {\left[\sum_{\beta, \alpha} R^{B^{\prime}}(E \mid \beta) R_{\mathcal{E}}^{S}(\beta \mid \alpha) R^{B}(\alpha \mid w)\right] } \\
& =\sum_{\beta, \alpha} R^{B^{\prime}}(E \mid \beta) R_{\mathcal{E}}^{S}(\beta \mid \alpha) R^{B}(\alpha \mid w)-\frac{1}{d} \operatorname{tr}(E) \\
& =\sum_{\beta, \alpha}\left(\Delta R^{B^{\prime}}(E \mid \beta)+\frac{1}{d} \operatorname{tr}(E)\right)\left(\Delta R_{\mathcal{E}}^{S}(\beta \mid \alpha)+\frac{1}{d^{2}}\right)\left(\Delta R^{B}(\alpha \mid w)+\frac{1}{d^{2}}\right)-\frac{1}{d} \operatorname{tr}(E) .
\end{aligned}
$$

We multiply out the parenthetical factors and note that every term that contains exactly one or two factors of the form $\Delta R$ will be found to be zero once the summations over phase space are implemented. This is due to the following equations that hold true because the normalization of the $R$ 's will always be equal to that of the $Q$ 's:

$$
\begin{aligned}
& \sum_{\alpha} \Delta R^{B}(\alpha \mid w)=0, \\
& \sum_{\alpha} \Delta R_{\mathcal{E}}^{S}(\beta \mid \alpha)=\sum_{\beta} \Delta R_{\mathcal{E}}^{S}(\beta \mid \alpha)=0, \\
& \sum_{\beta} \Delta R^{B^{\prime}}(E \mid \beta)=0 .
\end{aligned}
$$

Any term with fewer than the maximal possible number of $\Delta R$ 's will always have a factor of $\Delta R$ that is dependent on an unpaired phase space point having no match in the same term. For example, $\sum_{\beta, \alpha} \frac{1}{d^{2}} \Delta R^{B^{\prime}}(E \mid \beta) \Delta R_{\mathcal{E}}^{S}(\beta \mid \alpha)$ has an unpaired $\alpha$ so the sum over $\alpha$ yields zero. The two constant terms with values $\pm \frac{1}{d} \operatorname{tr}(E)$ cancel and the relationship we sought follows. Similar reasoning leads to the analogous equations when there is no channel or multiple ones.

\section{Interpreting $R^{B}$ in terms of the single- particle phase space}

Our aim here is to interpret $R^{B}\left(\alpha_{1} \mid w_{1}\right)$, in which $B$ is a striation in the large phase space $\mathbb{F}_{d}^{2}$, in terms of the smaller phase space $\mathbb{F}_{r}^{2}$.
In Section 7, we have shown that the $\Delta R^{B}\left(\alpha_{1} \mid w_{1}\right)$ 's are related to $\Delta Q\left(\alpha_{1} \mid w_{1}\right)$ by an analog of our standard formula (Eq. (124)). We now show that for certain striations $B, \Delta R^{B}\left(\alpha_{1} \mid w_{1}\right)$ is equal to $\Delta R^{B_{1}}\left(\alpha_{1} \mid w_{1}\right)$ for a corresponding striation $B_{1}$ of the smaller phase space, and that for all other striations, $\Delta R^{B}\left(\alpha_{1} \mid w_{1}\right)$ is zero.

From the definitions of Section 7 , we can write

$$
R^{B}\left(\alpha_{1} \mid w_{1}\right)=\frac{1}{d} \sum_{\alpha_{2}, \ldots, \alpha_{n}} \sum_{\beta \in \ell} Q(\alpha+\beta \mid w),
$$

where $\ell$ is the ray of the striation $B$, that is, the line in $B$ that passes through the origin. Doing the sum over $\alpha_{2}, \ldots, \alpha_{n}$, we get

$$
R^{B}\left(\alpha_{1} \mid w_{1}\right)=\frac{1}{d} \sum_{\beta \in \ell} Q\left(\alpha_{1}+\beta_{1} \mid w_{1}\right),
$$

where $\beta_{1}$ is the ordered pair $\left(\beta_{q 1}, \beta_{p 1}\right)$, the two components being the coefficients of $e_{1}$ and $\tilde{e}_{1}$, respectively, in the expansions of $\beta_{q}$ and $\beta_{p}$.

Let $m \in \mathbb{F}_{d}$ be the slope of the striation $B$ in the large phase space - assuming for now that the slope is not infinite - and consider the equation of the ray $\ell$ :

$$
\beta_{p}=m \beta_{q} .
$$

Taking the first-particle part of each side, we get

$$
\beta_{p 1}=\operatorname{Tr}\left(e_{1} \beta_{p}\right)=\operatorname{Tr}\left(e_{1} m \beta_{q}\right) .
$$

Now suppose $m$ is of the form $m=m_{1} e_{1}^{-1} \tilde{e}_{1}$, where $m_{1} \in \mathbb{F}_{r}$. Then $\operatorname{Tr}\left(e_{1} m \beta_{q}\right)$ is equal to $m_{1} \beta_{q 1}$, and we can write

$$
\beta_{p 1}=m_{1} \beta_{q 1} .
$$


This equation tells us that $\beta_{1}$ lies on the ray in $\mathbb{F}_{r}^{2}$ with slope $m_{1}$. Let us call this ray $\ell_{1}$ and the corresponding striation $B_{1}$. Thus if $m$ has the form $m=m_{1} e_{1}^{-1} \tilde{e}_{1}$, then as $\beta$ ranges over the ray $\ell$ in $\mathbb{F}_{d}^{2}, \beta_{1}$ ranges over the ray $\ell_{1}$ in $\mathbb{F}_{r}^{2}$. Moreover, it hits each point on this ray exactly $r^{n-1}$ times, since the other coefficients in the expansion of $\beta_{q}$ take $r^{n-1}$ values for each value of $\beta_{q 1}$.

Applying this result to Eq. (143), we have

$$
\begin{aligned}
R^{B}\left(\alpha_{1} \mid w_{1}\right) & =\frac{r^{n-1}}{d} \sum_{\beta_{1} \in \ell_{1}} Q\left(\alpha_{1}+\beta_{1} \mid w_{1}\right) \\
& =\frac{1}{r} \sum_{\beta_{1} \in \ell_{1}} Q\left(\alpha_{1}+\beta_{1} \mid w_{1}\right) .
\end{aligned}
$$

And this is exactly what we mean by $R^{B_{1}}\left(\alpha_{1} \mid w_{1}\right)$ for the density matrix $w_{1}$ which is the partial trace of $w$.

If the slope of $B$ is infinite, that is, if the ray $\ell$ is the solution to the equation

$$
\beta_{q}=0,
$$

then taking the first-particle part gives us

$$
\beta_{q 1}=0 .
$$

So again, the equation defines a ray in the smaller phase space, in this case the ray with infinite slope, and we can continue the argument as in the preceding paragraph.

The only other possibility is that $m=\sum_{j} m_{j} e_{1}^{-1} \tilde{e}_{j}$, with at least one of the $m_{j}$ 's, with $j \neq 1$, being nonzero. Let us suppose this is the case, and let $k \neq 1$ be such that $m_{k}$ is nonzero. Starting again with the equation (144) for the ray $\ell$ of slope $m$ in the large phase space, multiplying by $e_{1}$ and taking the trace, we get

$$
\beta_{p 1}=m_{1} \beta_{q 1}+m_{k} \beta_{q k}+\sum_{j \notin\{1, k\}} m_{j} \beta_{q j} .
$$

We claim that as $\beta$ ranges over $\ell$, the point $\left(\alpha_{q 1}, \alpha_{p 1}\right)$ in the small phase space takes all the values in $\mathbb{F}_{r}^{2}$ and takes each such value the same number of times. Suppose we first hold fixed every $\beta_{q j}$ with $j \notin\{1, k\}$ and let just $\beta_{q 1}$ and $\beta_{q k}$ vary. Because $m_{k}$ is nonzero, we can reach each value of $\beta_{p 1}$ for each value of $\beta_{q 1}$ : the $r^{2}$ values of $\left(\beta_{q 1}, \beta_{q k}\right)$ map one-to-one to the $r^{2}$ values of $\left(\beta_{q 1}, \beta_{p 1}\right)$. Moreover, this holds for every choice of the $\beta_{q j}$ 's with $j \notin\{1, k\}$. So as $\beta$ ranges over the whole ray, the ordered pair $\left(\beta_{q 1}, \beta_{p 1}\right)$ hits every point in the small phase space exactly $r^{n-2}$ times. Thus, for a striation with a slope of this form (that is, with some $m_{j} \neq 0$ with $j \neq 1$ ), the ray in the striation does not correspond to a ray in the small phase space. Rather, it covers the entire small phase space uniformly. It follows that the right-hand side of Eq. (143) does not depend on $\alpha_{1}$ at all; that is, $R^{B}\left(\alpha_{1} \mid w_{1}\right)$ is the uniform distribution. So $\Delta R^{B}\left(\alpha_{1} \mid w_{1}\right)$ is zero.

Thus, in the right-hand side of Eq. (124), we can get a nonzero contribution only for $r+1$ of the striations $B$, namely, those having slopes of the form $m=m_{1} e_{1}^{-1} \tilde{e}_{1}$ with $m_{1} \in \mathbb{F}_{r}$ together with the striation having infinite slope. For these cases, we can replace $\Delta R^{B}\left(\alpha_{1} \mid w_{1}\right)$ with $\Delta R^{B_{1}}\left(\alpha_{1} \mid w_{1}\right)$.

\section{Constraints on the $R$ distributions}

Every possible quantum state can be made out of pure states by convex combination. And every possible quantum operation can be made out of unitary channels, together with the operations of appending an auxiliary system and taking the partial trace. In this sense, if we give rules that define the possible pure states and the possible unitary transformations, then as long as we know how to do the required auxiliary manipulations, we have, in effect, specified the complete set of quantum states and quantum operations. Our aim in this Appendix is to give the rules that define pure states, unitary channels, and pure-state measurement outcomes when they are expressed in terms of our $R$ distributions. Since we know how to do a partial trace only when the dimension of the remaining system is an odd prime power, it is only for these cases that we claim to have a full specification of quantum states, operations, and measurements. Note that though we do not yet have a general way of appending an auxiliary system (because we do not yet have a general way of handing composite systems), when $r$ is an odd prime power we can use the partial trace to recognize a product of pure states with dimensions $r$ and $r^{2}$, and this is a sufficient starting point for our present purposes.

To express the rules for pure states and unitary transformations, it is very helpful to define the three-point structure function

$$
\Gamma_{\alpha \beta \gamma}=\frac{1}{d} \operatorname{tr}\left(A_{\alpha} A_{\beta} A_{\gamma}\right),
$$

where the $A$ 's are our phase-point operators. For the phase-point operators we have used in this paper (from Refs. $[24,34,35]), \Gamma$ has the following simple form:

$$
\Gamma_{\alpha \beta \gamma}=\frac{1}{d} \omega^{-2 \operatorname{Tr}_{\mathbb{F}_{d} / \mathbb{F}_{p}}(\langle\alpha, \beta\rangle+\langle\beta, \gamma\rangle+\langle\gamma, \alpha\rangle)},
$$

where $\langle\alpha, \beta\rangle$ is again the symplectic product

$$
\langle\alpha, \beta\rangle=\alpha_{p} \beta_{q}-\alpha_{q} \beta_{p} .
$$

In terms of $\Gamma$, we can recognize a pure state as a properly normalized Wigner function that satisfies [24]

$$
Q(\alpha \mid w)=\sum_{\beta, \gamma} \Gamma_{\alpha \beta \gamma} Q(\beta \mid w) Q(\gamma \mid w) .
$$


This condition comes from a familiar fact about a purestate density matrix $w: w^{2}=w$.

We can also express in terms of $\Gamma$ the condition for a unitary channel $U$. The transition quasiprobabilities $Q_{U}(\beta \mid \alpha)$, in addition to satisfying the normalization condition $\sum_{\beta} Q_{U}(\beta \mid \alpha)=1$, must also preserve $\Gamma$ in the sense that

$$
\sum_{\alpha, \beta, \gamma} Q_{U}\left(\alpha^{\prime} \mid \alpha\right) Q_{U}\left(\beta^{\prime} \mid \beta\right) Q_{U}\left(\gamma^{\prime} \mid \gamma\right) \Gamma_{\alpha \beta \gamma}=\Gamma_{\alpha^{\prime} \beta^{\prime} \gamma^{\prime}} .
$$

Though the proof in Ref. [24] that this condition is equivalent to unitarity was meant to apply only to the Wigner function defined in that paper, one finds that the proof applies equally well to the closely related definition proposed in Refs. [34] and [35], which we have used when $d$ is a power of an odd prime.

Eqs. (154) and (155) give us the conditions for a pure state and a unitary channel, respectively, in terms of the $Q$ distributions. To convert these equations into conditions on the $R$ distributions, we use the relations between $Q$ and $R$ obtained from Eqs. (37) and (54).

$$
Q(\alpha \mid w)=\sum_{B} R^{B}(\alpha \mid w)-\frac{1}{d}
$$

and

$$
Q(\beta \mid \alpha)=\frac{1}{\mathcal{Z}} \sum_{S} R^{S}(\beta \mid \alpha)-\frac{d^{2}-2}{d^{2}} .
$$

So we can say that the desired constraints on the $R$ distributions (for pure states and unitary channels) are Eqs. (154) and (155) with each $Q$ distribution replaced by the appropriate expression in Eq. (156) or (157).

As we note in the main text, these equations are not particularly simple. (Upon multiplying the factors out and collecting similar terms, one does not achieve any greater simplicity.) But it is conceivable that one can derive these equations from simpler principles more aptly framed for our epistemically restricted classical distributions.

Finally, we need to address the functions $R^{B}(E \mid \alpha)$. Again we start from the Wigner-function formulation. If the measurement outcome's POVM element $E$ is of the form

$$
E=|\psi\rangle\langle\psi|
$$

for some state vector $|\psi\rangle$, the quasiprobability function $Q(E \mid \alpha)$ must satisfy an equation analogous to Eq. (154):

$$
Q(E \mid \alpha)=\frac{1}{d} \sum_{\beta, \gamma} \Gamma_{\alpha \beta \gamma} Q(E \mid \beta) Q(E \mid \gamma) .
$$

We convert this equation to a condition on the $R$ 's via the analog of Eq. (156):

$$
Q(E \mid \alpha)=\sum_{B} R^{B}(E \mid \alpha)-1 .
$$

Suppose, then, that we have a number of sets $\left\{R^{B}\left(E_{j} \mid \alpha\right)\right\}$ of classical distributions, each of which satisfies Eqs. (159) and (160) (and thus represents a POVM element of the form (158)). Then any set of $R$ 's whose elements are of the form

$$
R^{B}(E \mid \alpha)=\sum_{j} c_{j} R^{B}\left(E_{j} \mid \alpha\right),
$$

where each $c_{j}$ is a positive real number and $\sum_{j} c_{j} \leq 1$, represents a legitimate outcome of a measurement, typically not associated with a pure state. Moreover, any legitimate outcome of a measurement can be obtained via Eq. (161), just as any mixed state can be obtained as a convex combination of pure states. (The difference here is that the $c_{j}$ 's can sum to a value less than one, since a POVM element can have any normalization less than one.) So Eqs. (159)-(161) together tell us what sets of $R$ functions are allowed in the specification of a measurement outcome.

\section{References}

[1] R. W. Spekkens, in Quantum Theory: Informational Foundations and Foils, edited by G. Chiribella and R. W. Spekkens (Springer, Dordrecht, 2016). doi:10.1007/978-94-017-7303-4_4.

[2] R. W. Spekkens, Phys. Rev. A 75, 032110 (2007). doi:10.1103/PhysRevA.75.032110.

[3] S. D. Bartlett, T. Rudolph, and R. W. Spekkens, Phys Rev. A 86, 012103 (2012). doi:10.1103/PhysRevA.86.012103.

[4] J. Larsson, AIP Conference Proceedings 1424, 211 (2012). doi:10.1063/1.3688973.

[5] A. J. Scott, J. Phys. A 41, 055308 (2008). doi:10.1088/1751-8113/41/5/055308.

[6] S. Mancini, V. I. Man'ko, P. Tombesi, Phys. Lett. A, 213, 1 (1996). doi:10.1016/0375-9601(96)00107-7.

[7] A. Ibort, V. I. Man'ko, G. Marmo, A. Simoni, F. Ventriglia, Phys. Scr. 79, 6, 065013 (2009). doi:10.1088/0031-8949/79/06/065013.

[8] L. Catani and D. E. Browne, New J. Phys. 19, 073035 (2017). doi:10.1088/1367-2630/aa781c.

[9] L. Catani and D. E. Browne, Phys. Rev. A 98, 052108 (2018). doi:10.1103/PhysRevA.98.052108.

[10] C. A. Fuchs and R. Schack, Rev. Mod. Phys. 85, 1693 (2013). doi:10.1103/RevModPhys.85.1693.

[11] O. Cohendet, P. Combe, M. Sirugue and S. M. Collin, J. Phys. A: Math. Gen. 21, 2875 (1988). doi:10.1088/0305-4470/21/13/012. 
[12] O. Cohendet, P. Combe and S. M. Collin, J. Phys. A: Math. Gen. 23, 2001 (1990). doi:10.1088/03054470/23/11/024.

[13] T. Hashimoto, M. Horibe and A. Hayashi, J. Phys. A: Math. Th. 40, 14253 (2007). doi:10.1088/1751$8113 / 40 / 47 / 015$.

[14] R. Raussendorf et al., Phys. Rev. A 101, 012350 (2020). doi:10.1103/PhysRevA.101.012350.

[15] P. Lillystone and J. Emerson, arXiv:1904.04268v1 [quant-ph] (2019).

[16] S. Kochen and E. P. Specker, J. Math. and Mech. 17, 59 (1967).

[17] J. S. Bell, Rev. Mod. Phys. 38, 447 (1966). doi:10.1103/RevModPhys.38.447.

[18] M. Zurel, C. Okay and R. Raussendorf, Phys. Rev. Lett. 125, $260404 \quad$ (2020). doi:10.1103/PhysRevLett.125.260404.

[19] M. Zurel, C. Okay, R. Raussendorf, and A. Heimendahl, arXiv2110.12318 [quant-ph] (2021).

[20] N. Harrigan and R. W. Spekkens, Found. Phys. 40, 125 (2010). doi:10.1007/s10701-009-9347-0.

[21] R. W. Spekkens, Phys. Rev. A 71, 052108 (2005). doi:10.1103/PhysRevA.71.052108.

[22] F. A. Buot, Phys. Rev. B 103700 (1974). doi:10.1103/PhysRevB.10.3700.

[23] J. H. Hannay and M. V. Berry, Physica D 1, 267 (1980). doi:10.1016/0167-2789(80)90026-3.

[24] W. K. Wootters, Ann. Phys. 176, 1 (1987). doi:10.1016/0003-4916(87)90176-X.

[25] D. Galetti and A. F. R. de Toledo Piza, Physica A 149267 (1988). doi:10.1016/0378-4371(88)90219-1.

[26] U. Leonhardt, Phys. Rev. Lett. 74, 4101 (1995). doi:10.1103/PhysRevLett.74.4101.

[27] U. Leonhardt, Phys. Rev. A 53, 2998 (1996). doi:10.1103/PhysRevA.53.2904.

[28] A. Vourdas, J. Phys. A: Math. Gen. 29, 4275 (1996). doi:10.1088/0305-4470/29/14/043.

[29] A. Luis, J. Perina, J. Phys. A: Math. Gen. 311423 (1998). doi:10.1088/0305-4470/31/5/012.

[30] T. Hakioglu, J. Phys. A: Math. Gen. 316975 (1998). doi:10.1088/0305-4470/31/33/008.

[31] A. Rivas, A. M. Ozorio de Almeida, Ann. Phys. 276123 (1999). doi:10.1006/aphy.1999.5942.

[32] K. S. Gibbons, M. J. Hoffman and W. K. Wootters, Phys. Rev. A 70, 062101 (2004). doi:10.1103/PhysRevA.70.062101.

[33] A. Vourdas, Rep. Prog. Phys. 67, 267 (2004). doi:10.1088/0034-4885/67/3/R03.
[34] A. B. Klimov and C. Muñoz, J. Opt. B: Quantum Semiclass. Opt. 7, S588 (2005). doi:10.1088/14644266/7/12/022.

[35] A. Vourdas, J. Phys. A: Math. Gen. 38, 8453 (2005). doi:10.1088/0305-4470/38/39/011.

[36] A. O. Pittenger and M. H. Rubin, J. Phys. A: Math. Gen. 38, 6005 (2005). doi:10.1088/0305$4470 / 38 / 26 / 012$.

[37] S. Chaturvedi, E. Ercolessi, G. Marmo, G. Morandi, N. Mukunda and R. Simon, J. Phys. A: Math. Gen. 39, 1405 (2006). doi:10.1088/03054470/39/6/014.

[38] D. Gross, J. Math. Phys. 47, 122107 (2006). doi:0.1063/1.2393152.

[39] D. Gross, Appl. Phys. B 86, 367 (2007). doi:10.1007/s00340-006-2510-9.

[40] S. Chaturvedi, N. Mukunda and R. Simon, J. Phys. A: Math. Th. 43, 075302 (2010). doi:10.1088/1751-8113/43/7/075302.

[41] A. Vourdas, J. Phys. A: Math. Th. 46, 043001 (2013). doi:10.1088/1751-8113/46/4/043001.

[42] K. Blum, Density Matrix Theory and Applications, 3rd edition (Springer, 2012). doi:10.1007/978-3-64220561-3.

[43] M. Ruzzi and D. Galetti, J. Phys. A: Math. Gen. 33, 1065 (2000). doi:10.1088/0305-4470/33/5/317.

[44] H. Zhu, Phys. Rev. Lett. 117, 120404 (2016). doi:10.1103/PhysRevLett.117.120404.

[45] W. F. Braasch Jr. and W. K. Wootters, Phys. Rev. A 102, 052204 (2020). doi:10.1103/PhysRevA.102.052204.

[46] M. Neuhauser, J. Lie Theory 12, 15 (2002).

[47] D. M. Appleby, J. Math. Phys. 46, 052107 (2005). doi:10.1063/1.1896384.

[48] H. F. Chau, IEEE Trans. Inf. Theory 51, 1451 (2005). doi:10.1109/TIT.2005.844076.

[49] W. K. Wootters and B. D. Fields, Ann. Phys. 191, 363 (1989). doi:10.1016/0003-4916(89)90322-9.

[50] D. Petz, K. M. Hangos, and A. Magyar, J. Phys. A: Math. Theor. 40, 7955 (2007). doi:10.1088/1751$8113 / 40 / 28 / \mathrm{S} 06$.

[51] D. W. Leung, PhD thesis, Stanford University (2000); arxiv:cs/0012017.

[52] G. M. D'Ariano and J. Lo Presti, Phys. Rev. Lett. 86, 4195 (2001). doi:10.1103/PhysRevLett.86.4195.

[53] W. Dür and J. I. Cirac, Phys. Rev. A 64, 012317 (2001). doi:10.1103/PhysRevA.64.012317. 
[54] D. W. Leung, J. Math. Phys. 44, 528 (2003). doi:10.1063/1.1518554.

[55] J. B. Altepeter, D. Branning, E. Jeffrey, T. C. Wei, P. G. Kwiat, R. T. Thew, J. L. O'Brien, M. A. Nielsen, and A. G. White, Phys. Rev. Lett. 90, 193601 (2003). doi:10.1103/PhysRevLett.90.193601.

[56] R. Lidl and H. Niederreiter, Introduction to finite fields and their applications, 2nd ed. (Cambridge Univ. Press, Cambridge 1994). doi:10.1017/CBO9781139172769.

[57] W. F. Braasch Jr. and W. K. Wootters, Entropy 24, 137 (2022). doi:10.3390/e24010137.

[58] V. Veitch, C. Ferrie, D. Gross, and J. Emerson, New J. Phys. 14 113011, (2012). doi:10.1088/13672630/14/11/113011.

[59] N. Delfosse, P. A. Guerin, J. Bian, and R. Raussendorf, Phys. Rev. X 5, 021003 (2015). doi:10.1103/PhysRevX.5.021003.

[60] R. Raussendorf, D. E. Browne, N. Delfosse, C. Okay, and J. Bermejo-Vega, Phys. Rev. A 95, 052334 (2017). doi:10.1103/PhysRevA.95.052334.

[61] M. S. Leifer, Quanta 3, 1 (2014). doi:10.12743/quanta.v3i1.22. 